\title{
Optimal Torque Split Strategy of Dual-Motor Electric Vehicle Using Adaptive Nonlinear Particle Swarm Optimization
}

\author{
Qingxing Zheng $\mathbb{D}^{1,2,3,4}$ Shaopeng Tian $\mathbb{D}^{1,2,3,4}$ and Qian Zhang $\mathbb{D}^{1,2,3,4}$ \\ ${ }^{1}$ School of Automotive Engineering, Wuhan University of Technology, Wuhan 430070, China \\ ${ }^{2}$ Hubei Key Laboratory of Advanced Technology for Automotive Components, Wuhan University of Technology, \\ Wuhan 430070, China \\ ${ }^{3}$ Hubei Collaborative Innovation Center for Automotive Components Technology, Wuhan 430070, China \\ ${ }^{4}$ Hubei Research Center for New Energy \& Intelligent Connected Vehicle, Wuhan University of Technology, \\ Wuhan 430070, China
}

Correspondence should be addressed to Shaopeng Tian; tianshp@whut.edu.cn

Received 16 January 2020; Revised 30 March 2020; Accepted 7 April 2020; Published 21 May 2020

Guest Editor: Chi-Hua Chen

Copyright (c) 2020 Qingxing Zheng et al. This is an open access article distributed under the Creative Commons Attribution License, which permits unrestricted use, distribution, and reproduction in any medium, provided the original work is properly cited.

\begin{abstract}
In order to exploit the potential of energy saving of dual-motor powertrain over single-motor powertrain, this paper proposes a time-efficient optimal torque split strategy for a front-and-rear-axle dual-motor electric powertrain. Firstly, a physical model of electric vehicle powertrain is established in Matlab/Simulink platform and further validated by real-vehicle experiments. Subsequently, a three-layer energy management strategy composed of demanded torque calculation layer, mode decision layer, and torque split layer is devised to enhance the total operating efficiency of two motors. Specifically, the optimal torque split strategy using adaptive nonlinear particle swarm optimization (ANLPSO) is embedded in the torque split layer. Finally, two conventional strategies (even distributed strategy and rule-based strategy) for dual-motor powertrain are considered for comparison to verify the efficacy of the proposed strategy. Tremendous results demonstrate that the dual-motor powertrain with this proposed optimal torque split strategy develops energy saving by $11.88 \%$ and $12.18 \%$ against single-motor powertrain in the NEDC and WLTP. Compared to two conventional torque split strategies, it is able to reduce the total motor loss by $12.17 \%$ and $8.1 \%$ in NEDC and $11.91 \%$ and $8.07 \%$ in WLTP, respectively, which indicates the prominent optimization performance and a great potential in realistic applications.
\end{abstract}

\section{Introduction}

To relieve increasing burden caused by environmental pollution and energy shortage, electric vehicles (EVs) emerged as a promising approach all over the world [1]. While the energy efficiency of internal combustion engine (ICE) vehicles is merely from $15 \%$ to $20 \%$, typical EVs utilize $50-75 \%$ of the energy effectively to propel the vehicle $[2,3]$. There are three main categories of EVs, including hybrid electric vehicles (HEVs), fuel cell electric vehicles (FCHEVs), and battery electric vehicles (BEVs) [4], among which BEVs have the best "tank-to-wheel" efficiency [5]. Furthermore, the salient upsides of no $\mathrm{CO}_{2}$ emissions and other pollutants lead BEVs to be one of the most promising alternatives for green transportation and sustainable future mobility [6]. However, BEVs are still facing driving range concern, mainly owing to the long charging time and unsatisfactory energy density of power battery when compared to gasoline and diesel [7].

1.1. Literature Review. To improve the driving range of the conventional single-motor-driven BEVs, from the perspective of powertrain topology and control, various powertrain structures and relevant control strategies have been proposed and investigated. These advanced configurations offer additional degrees of freedom to achieve more efficient torque distribution and independent control of each driving 
shaft/wheel. The literature review mainly focuses on two following aspects: powertrain topology and energy management strategy.

1.1.1. Powertrain Topology. According to the number of motors used in the vehicle and their configuration, existing powertrain topology of BEVs can be classified into two types including the centralized single-motor-driven powertrain and the distributed multimotor-driven powertrain.

The centralized single-motor one-speed-driven powertrain is the most common structure in modern BEVs. As the motor substitutes the ICE to propel the vehicle through a one-speed reducer, it can be easily installed on the conventional chassis without excessive modification. Compared to one-motor multispeed powertrain, this kind of powertrain is known as the simplest structure, whereas it would increase the working burden of both motor and controller. Moreover, it has relatively low energy efficiency [8]. To address such drawbacks, some researchers proposed the single-motor with multispeed gearbox powertrain. The results indicated that multispeed gearbox could downsize the motor and improve the overall energy efficiency by about $5-12 \%$ through the improvement of motor operating points $[9,10]$. However, this powertrain is rarely used in practice because the additional multispeed gearbox might cause the increase in cost and control difficulty of shift smoothness (especially with the increase in gears) [11].

Recently, some researchers have focused on distributed multimotor-driven powertrain. Existing multimotor-driven powertrain is mainly classified into three categories [12]: dual-motor powertrain, triple-motor powertrain (shown in Figure 1(c)) [13], and four-motor powertrain (shown in Figure 1(d)) [14-16]. Specially, dual-motor powertrain has been actively investigated as a promising alternative to single-motor powertrain. Hu et al. studied a novel dualmotor powertrain in which the speed of two motors is coupled through planetary gear and the torque is coupled through shaft-fixed gear. The simulation shows that this powertrain could improve energy efficiency effectively [17]. However, the speed and torque coupling devices increase structure complexity, manufacturing cost, and control difficulty. Gao et al. researched a practical dual-motor coaxial powertrain for battery electric buses with dynamic programming (DP) method, which could eliminate power interruption and realize a great reduction of energy consumption [18]. Actually, it is one kind of planet gearbased coupling system, and additional coupling devices, such as clutches and synchronizers, are still needed, which burden the mechanism design and control. In previous study [19], there is another type of powertrain with two wheel-hub motors directly driving the left/right wheel through a onespeed reducer, shown in Figure 1(b). But this powertrain is rarely used in practice. Ruan and Song devised a novel dualmotor two-speed powertrain as one of the parallel direct drive layouts for BEVs. This structure could achieve a great efficiency improvement and smooth shifting process without the increase in mechanical complexity [20]. Kang et al. proposed an electric powertrain with the front and rear shaft driven by two motors, respectively (shown in Figure 1(a)). It exhibits great enhancement of drivability and potential of energy saving [21]. Due to the low manufacturing cost, simple architecture, energy efficiency improvement potential, and great dynamics performance, the front-and-rearaxle dual-motor powertrain is investigated in this paper.

1.1.2. Energy Management Strategy. In dual-motor powertrain, energy management strategy plays an important role in power split among two motors and energy saving. $\mathrm{Hu}$ et al. proposed the multimode control strategy and power split strategy for a dual-motor powertrain with a planetary gear. The results show great improvement in economic performance and driving range [18]. Urbina Coronado et al. designed a two-parameter rule-based control strategy for a dual-motor powertrain. The results indicate that a combination of high-speed transition threshold and high torque transition threshold could achieve the best results in energy saving and drivability [22]. In previous study [23], theoretical solutions of power loss optimization were conducted for a front-rear-induction-motor EVs. It is confirmed that single-motor mode takes priority over dual-motor mode under required low torque condition, while even torque distribution is the optimal control in dual-motor mode under required middle or high torque condition. Yuan and Wang investigated the optimal torque distribution strategy for an EV with two identical PMSMs to improve motor efficiency in the region of low torque and high speed. This study reveals that the motor efficiency could be significantly improved in low torque region when one motor is operating and the other motor is fully turned off through a clutch. And in the region of middle or high torque, the required torque should be equally distributed to minimize power loss when only the longitudinal dynamics is considered [24]. Wang et al. suggested a mode decision system based on fuzzy logic for a dual-motor powertrain. The frequent mode switching is reduced by the defined offset and compensation for mode switching line [25]. Zhao et al. developed a novel rule-based strategy extracted from DP optimization results. The simulation results show that the improved strategy could reach 95\% energy-saving effect of that under DP [26].

1.2. Motivation and Innovation. This study was carried out in order to prove the potential of energy efficiency improvement of a front-and-rear-axle dual-motor powertrain over single-motor powertrain. In aforementioned literature, tremendous research studies were conducted on control strategy for front-and-rear-axle-driven powertrain. It is concluded that the switching threshold between singlemotor mode and dual-motor mode is related to motor speed. In practice, the motor speed varies with unexpected road conditions, which requires the switching threshold and torque split strategy to be optimized in real time for better control. However, a vast majority of existing torque split strategies are designed based on theoretical analysis or empirical rules, using a fixed torque switching threshold for control simplicity but with the sacrifice of optimality. 


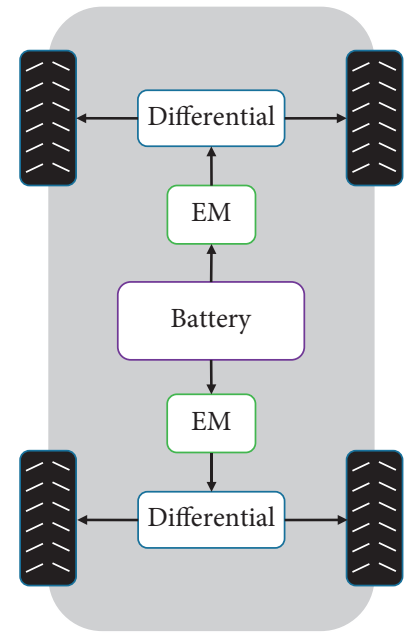

(a)

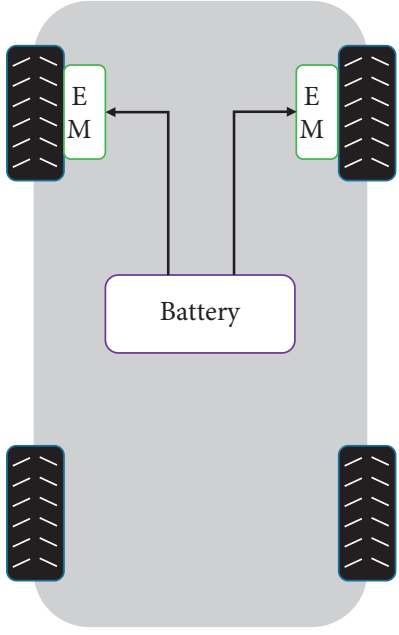

(b)

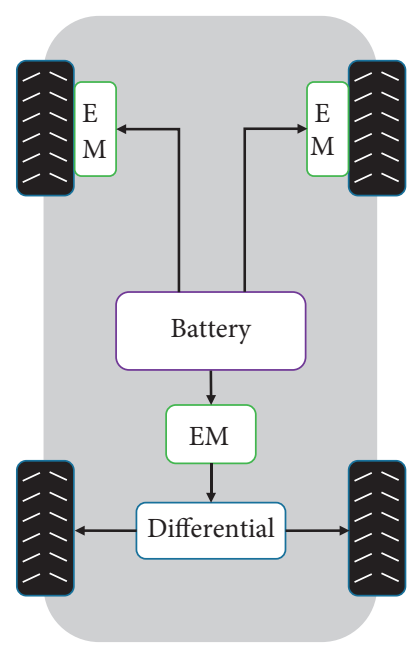

(c)

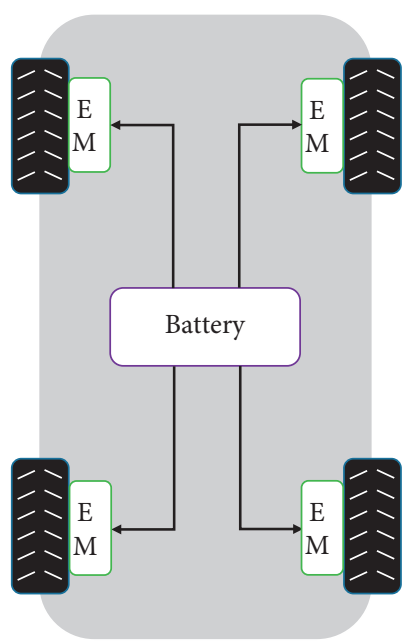

(d)

FIgURE 1: BEV powertrains.

To address this research gap, this paper developed a time-efficient optimal three-layer energy management strategy based on adaptive nonlinear particle swarm optimization (ANLPSO). The main contributions incorporate three aspects. Firstly, the optimal torque split strategy was established to search the best torque split ratio of two motors instantaneously and thus achieve the optimal energy efficiency. Secondly, the algorithm architecture and main influencing factors of PSO were improved to construct the proposed ANLPSO, which could perform a tradeoff between optimality and real-time applicability. Eventually, a comprehensive comparison was conducted between two common torque split strategies and the optimal one.

The organization of this paper is as follows: the powertrain architecture is described and modeled in Section 2. The three-layer energy management strategy and the realtime optimal torque distribution strategy are designed in Section 3. In Section 4, the physical model is validated by real-vehicle experiment, followed by the comparison and analysis of the dual-motor powertrain simulation results between even distribution strategy, the rule-based strategy in literature, and the proposed real-time optimal control strategy. Finally, conclusions are summarized in Section 5.

\section{Powertrain Architecture and Model}

2.1. Electric Powertrain Configuration. Figure 1(a) illustrates the configuration of the front-and-rear-axle dual-motor electric powertrain. In this architecture, two identical PMSMs are coupled to the front axle or the rear axle through a one-speed gear set and a differential mechanism, respectively. The target dual-motor BEV in this paper is a commercial vehicle prototype (shown in Figure 2), with all relevant parameters detailed in Tables 1-3.

2.2. Vehicle Longitudinal Dynamics Model. This research focuses on the energy efficiency of the dual-motor powertrain. Thus, only the longitudinal dynamics vehicle model is studied in this paper because the vehicle vertical vibration and vehicle yaw have little influences on energy efficiency. Generally, the overall resistance force over wheels consists of air resistance, rolling resistance, and climbing resistance. However, in this study, the climbing resistance is neglected since the driving cycles are horizonal. Hence, the final resistance force over wheels is calculated by equations shown below:

$$
\begin{aligned}
F_{r} & =\frac{C_{d} A v(k)^{2}}{21.15}+m g C_{r}+\frac{J_{t} a(k)}{r^{2}}, \\
J_{t} & =J_{v}+J_{w}+\left(J_{\mathrm{EM} 1}+J_{\mathrm{EM} 2}\right) i_{t}^{2}, \\
m \frac{\mathrm{d} v(k)}{\mathrm{d} t} & =\frac{\left(T_{\mathrm{EM} 1}+T_{\mathrm{EM} 2}\right) i_{t} \eta_{t}}{r}-F_{r},
\end{aligned}
$$

where $F_{r}$ is the overall resistance force over wheels; $C_{d}$ is the air drag coefficient; $A$ is the frontal area of vehicle; $v$ is the velocity of vehicle; $k$ is the $k$ th time step; $m$ is the mass of vehicle; $g$ is the gravitational acceleration; $C_{r}$ is the rolling resistance coefficient; $a$ is the longitudinal acceleration of vehicle; $J_{t}$ is the total rotary inertia at the wheels; $J_{v}$ is the rotary inertia of vehicle chassis; $J_{w}$ is the total rotary inertia of four wheels; $J_{\mathrm{EM} 1}$ and $J_{\mathrm{EM} 2}$ are the rotary inertia of EM1 and $\mathrm{EM} 2$, respectively; $T_{\mathrm{EM} 1}$ and $T_{\mathrm{EM} 2}$ are the output torque of EM1 and EM2, respectively; $i_{t}$ is the transmission ratio; $\eta_{t}$ is the transmission efficiency; and $r$ is the rolling radius of wheel.

2.3. Driver Model. The driver model is designed based on feedforward-feedback PI controller. Based on the bias between the desired velocity (given by driving cycle) and the actual velocity, acceleration or braking command will be given by the driver model. To improve the control precision and reduce the fluctuation of driver commands, a feedforward control module based on desired velocity, a feedback control module based on driver commands, and an 


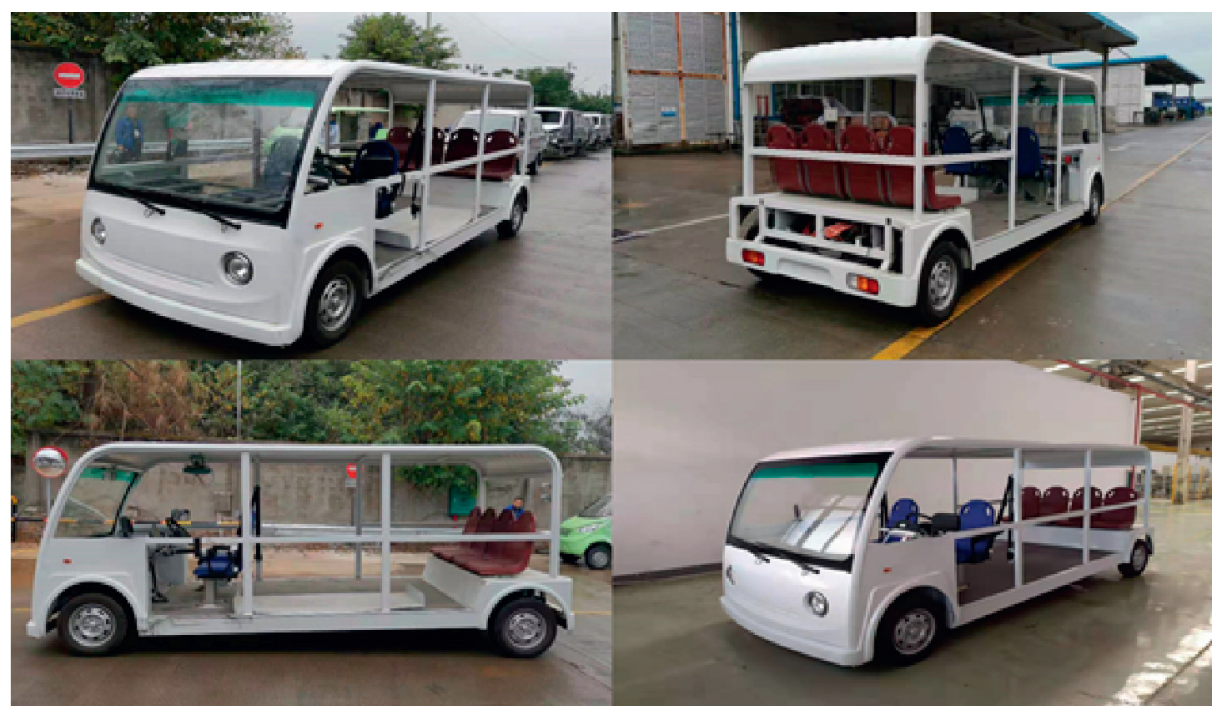

Figure 2: Target dual-motor BEV.

TABLE 1: Vehicle parameters of target vehicle.

\begin{tabular}{lc}
\hline \multicolumn{2}{c}{ Vehicle characteristics } \\
\hline Vehicle mass (half load) $(m)$ & $2000 \mathrm{~kg}$ \\
Dynamic rolling radius $(r)$ & $0.273 \mathrm{~m}$ \\
Frontal area $(A)$ & $5 \mathrm{~m}^{2}$ \\
Transmission efficiency $\left(\eta_{t}\right)$ & 0.9504 \\
Air drag coefficient $\left(C_{d}\right)$ & 0.4 \\
Rolling resistance coefficient $\left(C_{r}\right)$ & 0.01 \\
Rotary mass coefficient $\left(J_{t}\right)$ & 1.12 \\
Gravitational acceleration $(g)$ & $9.8 \mathrm{~m} / \mathrm{s}^{2}$ \\
Final drive ratio (front $/ \mathrm{rear}$ axle) & 6.732 \\
Top speed & $85 \mathrm{~km} / \mathrm{h}$ \\
Climbing capacity & $20 \%$ at $25 \mathrm{~km} / \mathrm{h}$ \\
Acceleration time $(0-50 \mathrm{~km} / \mathrm{h})$ & $7 \mathrm{~s}$ \\
\hline
\end{tabular}

additional high-pass filter were added. The driver command can be calculated by the following equation:

$$
\begin{gathered}
\operatorname{Acc}_{\mathrm{pdl}}(k)=K_{F F} V_{d}(k)+K_{p}\left(V_{d}(k)-V_{a}(k)\right) \\
+\int_{0}^{k}\left(K_{i}\left(V_{d}(k)-V_{a}(k)\right)+K_{F B} D_{c}\right) \mathrm{d} k \\
V_{d}(k)-V_{a}(k) \geq 0 \\
\operatorname{Brk}_{\mathrm{prs}}(k)=G_{\mathrm{brk}} *\left(K_{F F} V_{d}(k)+K_{p}\left(V_{d}(k)-V_{a}(k)\right)\right. \\
\left.+\int_{0}^{k}\left(K_{i}\left(V_{d}(k)-V_{a}(k)\right)+K_{F B} D_{c}\right) \mathrm{d} k\right) \\
V_{d}(k)-V_{a}(k)<0
\end{gathered}
$$

where $A c c_{\mathrm{pdl}}$ is the acceleration pedal command; $G_{\text {brk }}, K_{F F}, K_{p}, K_{i}$, and $K_{F B}$ are constant gain of the demanded brake pressure, feedforward module,
TABle 2: Parameters of driving motor.

\begin{tabular}{lc}
\hline Name & Value \\
\hline Type & PMSM (ETG029) \\
Rated/peak power & $15 / 44.6 \mathrm{~kW}$ \\
Rated/peak torque & $31 / 117 \mathrm{Nm}$ \\
Maximum rotation speed & $7500 \mathrm{rpm}$ \\
Nominal voltage & $115 \mathrm{~V}$ \\
Peak efficiency & $96 \%$ \\
\hline
\end{tabular}

TABle 3: Parameters of power battery.

\begin{tabular}{lc}
\hline Name & Value \\
\hline Type & $\mathrm{LiFePO}_{4}$ \\
Capacity $(C)$ & $375 \mathrm{Ah}$ \\
Nominal voltage & $115.2 \mathrm{~V}$ \\
Operating temperature range & $-20-55^{\circ} \mathrm{C}$ \\
Mass & $320 \mathrm{~kg}$ \\
\hline
\end{tabular}

proportional gain, integral gain, and feedback module, respectively; $V_{d}(k)$ is the desired velocity; $V_{a}(k)$ is the actual velocity; $D_{c}$ is the driver command given by driver model; and $\mathrm{Brk}_{\mathrm{prs}}$ is the demanded brake pressure.

2.4. Driving Motor Model. The driving motors in this powertrain consist of two identical PMSMs, which can work as the driving motor to propel the vehicle or as a generator to recover braking energy. The data of motor external characteristics and efficiency characteristics are based on experimental data, which is shown in Figure 3. The output torque of motor is simplified as a function of motor speed and motor torque command, as shown in equation (6). The efficiency characteristics are related to motor speed and motor output torque, as shown in equation (7): 


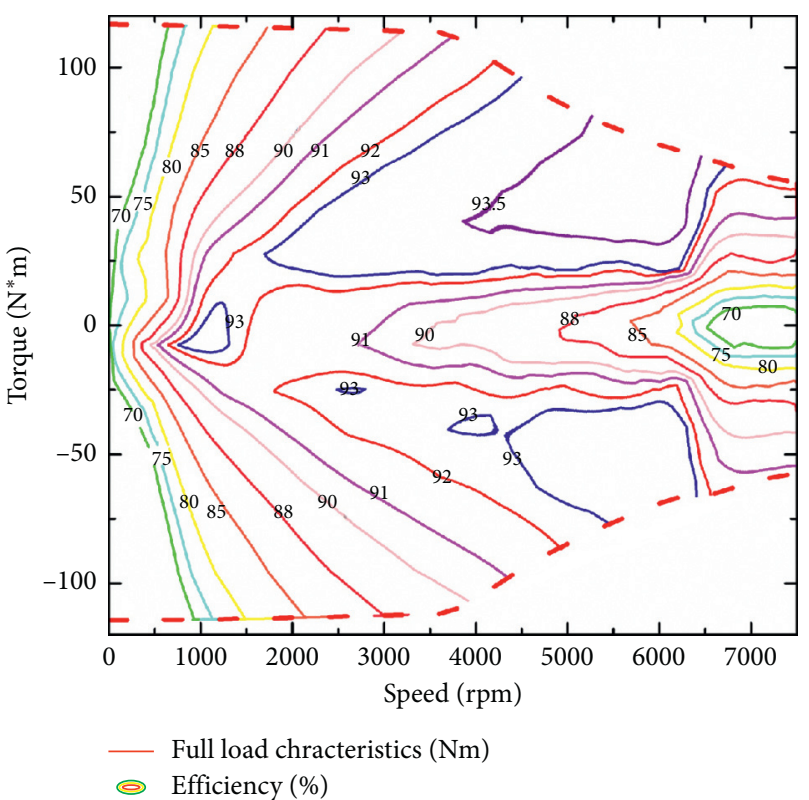

(a)

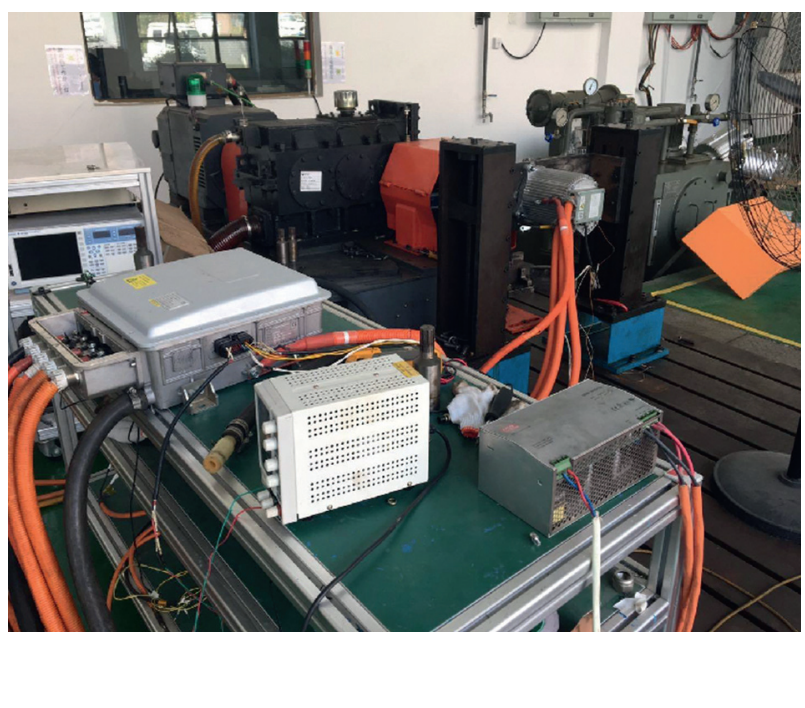

(b)

Figure 3: (a) External characteristics and efficiency characteristics; (b) motor test bench.

$T_{\mathrm{EM} i}(k)= \begin{cases}l_{\mathrm{EM} i}(k) \times T_{\operatorname{chgmaxEM} i}\left(n_{\mathrm{EM} i}(k)\right), & l_{\mathrm{EM} i}(k)<0 ; i=1,2, \\ l_{\mathrm{EM} i}(k) \times T_{\operatorname{dismaxEM} i}\left(n_{\mathrm{EM} i}(k)\right), & l_{\mathrm{EM} i}(k) \geq 0 ; i=1,2,\end{cases}$

$\eta_{\mathrm{EM} i}(k)=f\left(n_{\mathrm{EM} i}(k), T_{\mathrm{EM} i}(k)\right), \quad i=1,2$,

$P_{\mathrm{EM} i}(k)= \begin{cases}\frac{n_{\mathrm{EM} i}(k) T_{\mathrm{EM} i}(k)}{9550 \eta_{\mathrm{EM} i}(k)}, & T_{\mathrm{EM} i}(k) \geq 0 ; i=1,2, \\ \frac{n_{\mathrm{EM} i}(k) T_{\mathrm{EM} i}(k) \eta_{\mathrm{EM} i}(k)}{9550}, & T_{\mathrm{EM} i}(k)<0 ; i=1,2,\end{cases}$

where $n_{\mathrm{EM}}$ is the motor speed, $T_{\mathrm{EM}}$ is the output torque of motor, $l_{\mathrm{EM}}$ is the motor torque command, $T_{\text {chgmaxEM }}\left(n_{\mathrm{EM}}\right)$ is the maximum charge torque of motor at $n_{\mathrm{EM}}$, $T_{\text {dismaxEM }}\left(n_{\mathrm{EM}}\right)$ is the maximum discharge torque of motor at $n_{\mathrm{EM}}, \eta_{\mathrm{EM}}$ is the efficiency of motor, and $P_{\mathrm{EM}}$ is the electric power of motor.

2.5. Battery Model. The electrochemistry reaction inside the battery is quite complicated, which is not the scope of this study. Hence, the open equivalent circuit model [27] is adopted, which reflects the relationship between open circuit voltage, inner resistance, and the state of charge (SOC). In this case, when the battery output power is given, the current of battery and SOC can be calculated as follows:

$$
P_{b}(k)=\sum_{i=1}^{2} P_{\mathrm{EM} i}(k)+I(k)^{2} R(\operatorname{SOC}(k)),
$$

$$
I(k)=\frac{P_{b}(k)}{U_{\mathrm{OC}}(\operatorname{SOC}(k))},
$$

$$
\operatorname{SOC}(k)=\operatorname{SOC}_{\text {ini }}-\frac{\int_{0}^{k} I_{b}(k) \mathrm{d} k}{3600 C},
$$

where $P_{b}$ is the total battery power, $I$ is the battery current, $R(\operatorname{SOC}(k))$ is the internal resistance at $\operatorname{SOC}(k)$, $U_{\mathrm{OC}}(\operatorname{SOC}(k))$ is the open circuit voltage at $\operatorname{SOC}(k), \mathrm{SOC}_{\text {ini }}$ is the initial value of SOC, and $C$ is the battery capacity.

2.6. Driving Cycle. The driving cycle is a standard test procedure in which the desired velocity is fixed with respect to time. The driver is supposed to propel the vehicle to reach the desired velocity within a certain error tolerance range to ensure accuracy of the test or simulation. Numerous driving cycles are proposed to evaluate energy consumption, fuel consumption, and emissions for sorts of vehicles in different countries, such as New European Driving Cycle (NEDC), Urban Dynamometer Driving Schedule (UDDS), Japan Cycle 08 (JC08), World Light Vehicle Test Procedure (WLTP). In this research, NEDC and WLTP are selected as the standard test cycle for the target vehicle. As mentioned above, the target vehicle is a commercial sample vehicle with a maximum velocity of $85 \mathrm{~km} /$ h. Therefore, the portions with the velocity exceeding $85 \mathrm{~km} / \mathrm{h}$ in NEDC and WLTP are limited to $85 \mathrm{~km} / \mathrm{h}$. Figure 4 shows the driving cycles with velocity limitation. 


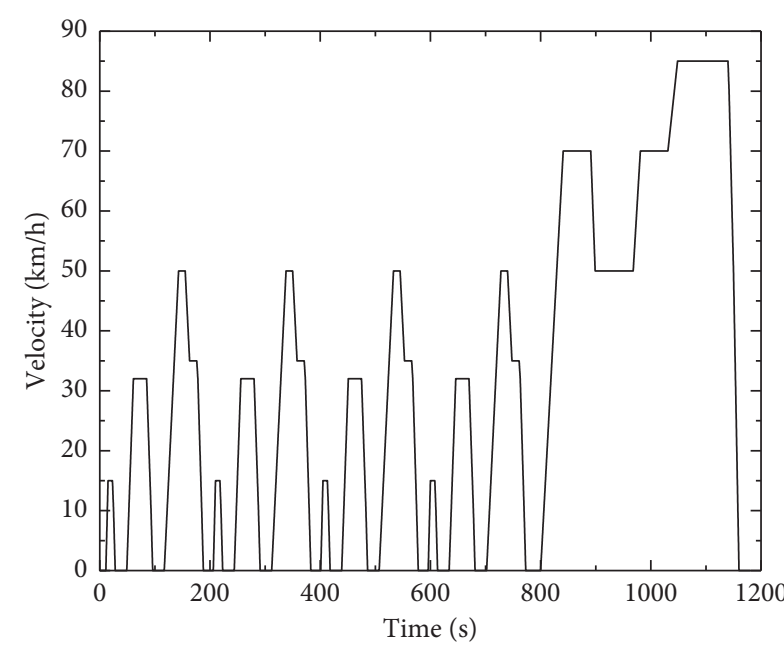

— Desired velocity

(a)

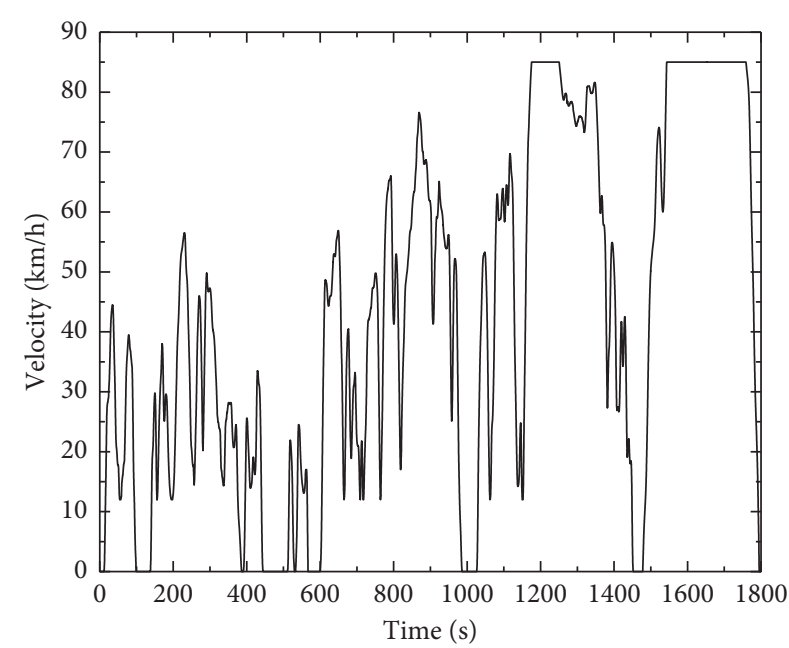

— Desired velocity

FIGURE 4: (a) NEDC with velocity limitation; (b) WLTP with velocity limitation.

\section{Optimal Energy Management Strategy}

To optimize energy efficiency and dynamics performance, energy management strategy plays a paramount role in power split for dual-motor powertrain. In this research, the optimal energy management strategy consists of three layers: demanded torque calculation (upper layer), vehicle operating mode decision (decision layer), and real-time optimal torque split (lower layer), with the schematic diagram shown in Figure 5.

3.1. Demanded Torque Calculation. In the upper layer, the demanded torque can be calculated based on the driver's command, state of two motors, and sensor signals of hydraulic brake. In detail, the demanded driving torque is determined by the acceleration pedal signal and the available maximum output torque of two motors, while the demanded brake pressure is decided by driver's brake command and constant gain of the demanded brake pressure (as mentioned in Section 2.3).

3.2. Vehicle Operating Mode Decision. The decision layer is designed to define the vehicle operating mode based on driving torque command, brake pressure, SOC of battery, and current velocity. The simple and robust "if-else" control strategy is adopted to yield the most appropriate operating mode for this powertrain through state machine. The mode switching logic is illustrated in Figure 6.

Electric mode: if the demanded driving torque is greater than zero and the battery SOC is greater than discharge lower limit (SOC_l), the vehicle is in electric mode. And the magnitude of the demanded driving torque determines whether the vehicle is driven by a single motor or two motors, which is further determined by the torque split strategy.
Regenerative brake mode: if the battery SOC is below charge upper limit (SOC_h) and the demanded brake torque is nonzero and not greater than the available maximum torque of two motors and the current velocity is greater than a fixed value, the vehicle will operate in the regenerative brake mode. In order to adapt to different braking requirements, the regenerative brake mode is further divided into three suboperating modes including energy recovery priority mode to maintain high regenerative braking efficiency, braking performance priority mode to obtain maximum braking force, and safety priority mode for the sake of safety in wet/snow road surface [28]. In this study, the energy recovery priority mode is the main working mode for regenerative braking.

Hydraulic brake mode: considering vehicle safety, whenever the demanded brake torque exceeds the threshold defined by the ECE safety regulation or the current velocity is smaller than a specified value, the hydraulic brake mode is switched on.

3.3. Real-Time Optimal Torque Split Strategy. The lower layer is torque split strategy, composed of the optimal driving torque split strategy and braking torque split strategy. However, in this paper, the proposed real-time optimal driving torque split strategy plays the major role and would be detailed further. At the same time, to explore its potential, the even distribution strategy and the control strategy proposed by some researchers would be included for comparison in terms of energy efficiency.

3.3.1. Baseline Driving Torque Split Strategy. For dual-motor powertrain, the simplest and most common torque split strategy is even distribution strategy, in which the demanded driving torque is always distributed equally to two motors to 


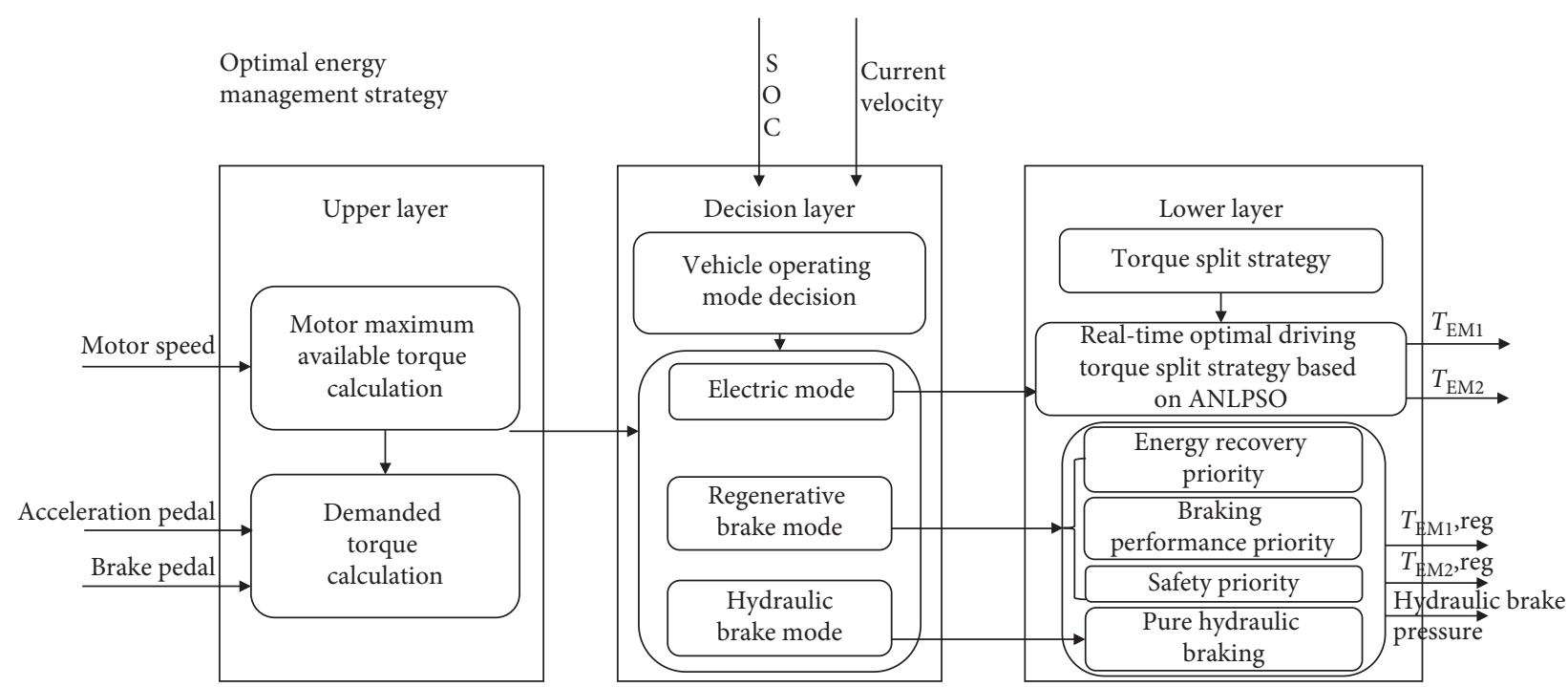

Figure 5: Schematic of optimal energy management strategy.

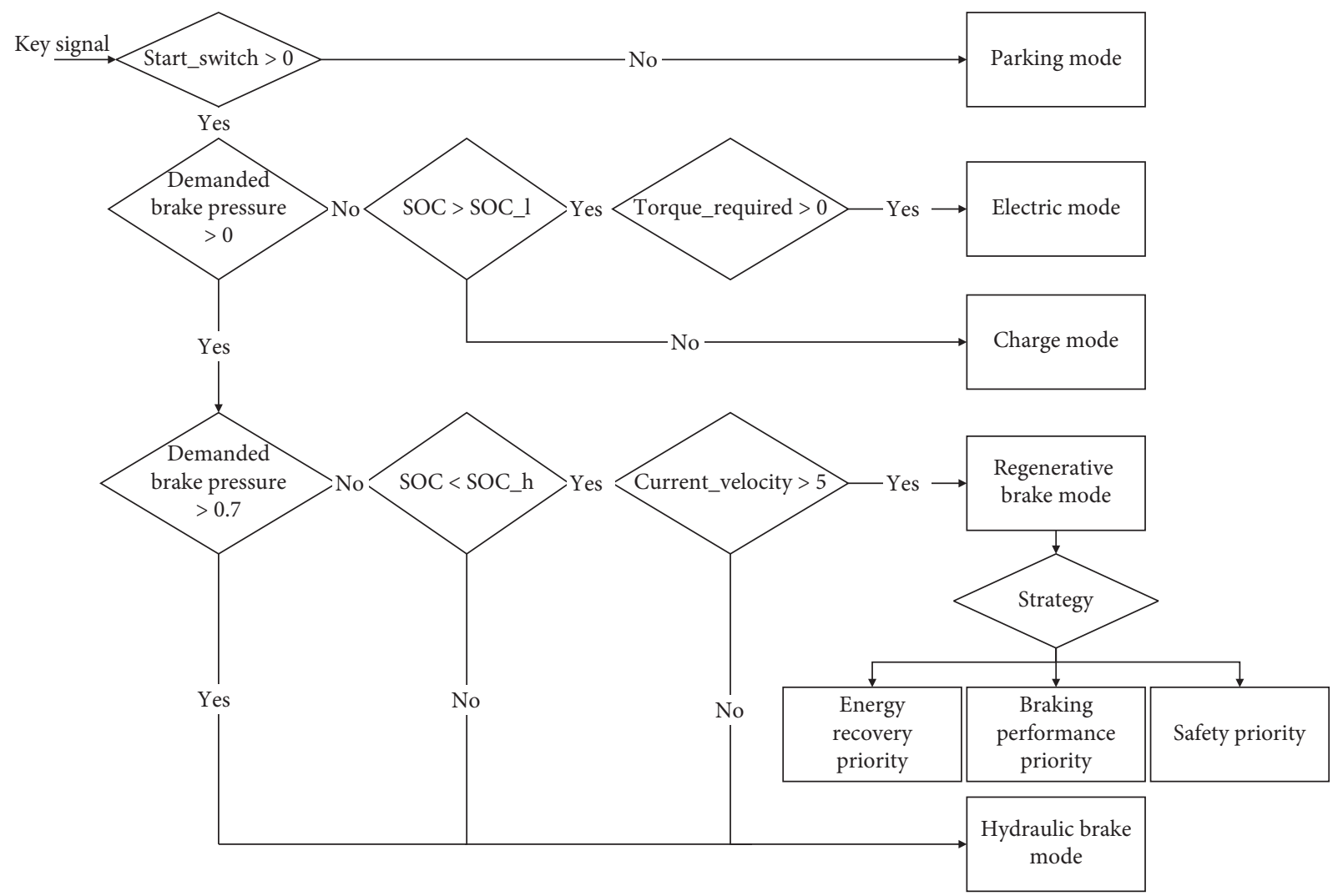

FIgURE 6: Mode switching logic.

propel the vehicle. Thus, even distribution strategy is defined as the baseline torque split strategy.

In the previous research, another critical strategy is the rule-based torque split strategy, shown in Figure 7 . If the demanded driving torque is smaller than a preset torque

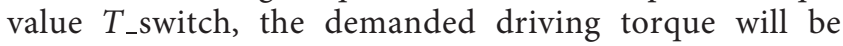
totally distributed to a single motor to propel the vehicle. Else, the driving torque is distributed equally to two motors. To define a proper torque threshold $T_{\text {_ }}$ switch, the optimization procedure is proceeded using the genetic

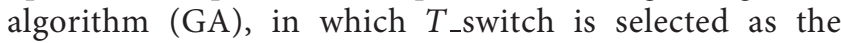
optimization variable and the energy consumption of typical cycles simulation is chosen as the optimization objective. The optimal T_switch $(102.6 \mathrm{Nm})$ is obtained after 100 iterations. As a contrast, it is also researched in this paper. 


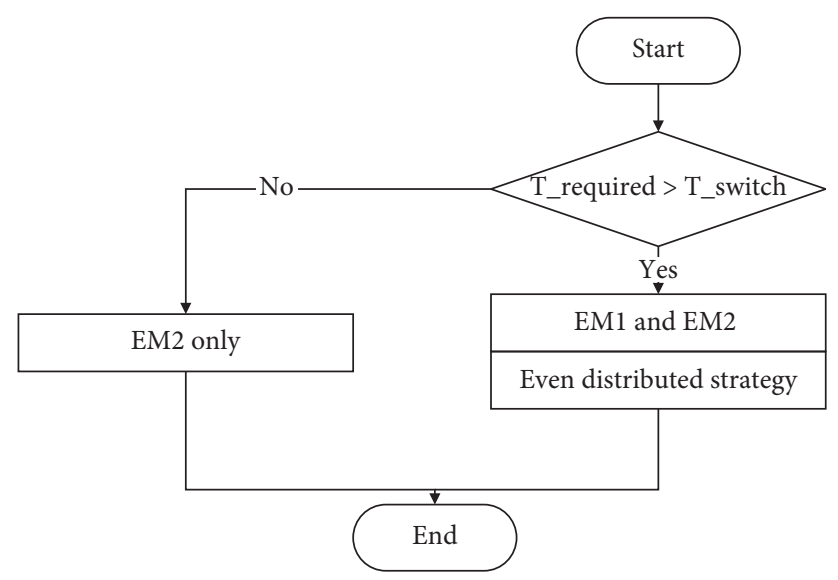

FIgURE 7: Rule-based torque split strategy.

\subsubsection{Real-Time Driving Torque Split Strategy Based on ANLPSO}

(1) Optimization problem description

Optimization Variables. The power split ratio is considered as the optimization variable, which is defined as the required power of front motor divided by the total demanded power:

$$
\begin{aligned}
\alpha(k) & =\frac{P_{\mathrm{EM} 2}(k)}{P_{d}(k)}=\frac{T_{\mathrm{EM} 2}(k) n_{\mathrm{EM} 2}(k)}{T_{\mathrm{EM} 1}(k) n_{\mathrm{EM} 1}(k)+T_{\mathrm{EM} 2}(k) n_{\mathrm{EM} 2}(k)} \\
& =\frac{T_{\mathrm{EM} 2}(k)}{T_{\mathrm{EM} 1}(k)+T_{\mathrm{EM} 2}(k)}=\frac{T_{\mathrm{EM} 2}(k)}{T_{d}(k)}
\end{aligned}
$$

where $\alpha$ is the power split ratio and $P_{d}$ and $T_{d}$ are the demanded power and torque of driver, respectively. According to Equation (12), since the powertrain architecture of the front axle is the same as that of rear axle, the motor speed of EM1 is the same as that of EM2. Thus, the power split ratio is the same as the torque split ratio. The optimized output torque of EM1 and EM2 can be given as

$$
\begin{aligned}
& T_{\mathrm{EM} 2}(k)=\alpha(k) T_{d}(k) ; \\
& T_{\mathrm{EM} 1}(k)=(1-\alpha(k)) T_{d}(k) .
\end{aligned}
$$

According to equations (12) and (13), it is obvious that the range of optimization variable is $0-1$. Because of the symmetrical front and rear powertrain architecture, the range of optimization variables can be simplified to $0-0.5$.

Constraints. The output torque of EM1 and EM2 is supposed not to exceed the available maximum discharge torque of motor. It is also nonnegative as the energy efficiency is lower when one motor drives the other one to generate electricity. In detail, it can be expressed as

$$
\begin{aligned}
& 0 \leq T_{\mathrm{EM} 1}(k) \leq T_{\text {dismaxEM1 }}\left(n_{\mathrm{EM} 1}(k)\right), \\
& 0 \leq T_{\mathrm{EM} 2}(k) \leq T_{\text {dismaxEM} 2}\left(n_{\mathrm{EM} 2}(k)\right) .
\end{aligned}
$$

Objective Function. For the given demanded driving torque and motor speed at time $k$, the main purpose of the optimal driving torque split strategy is to maximize total efficiency of two motors. Hence, the total efficiency is used as objective function, which can be formulated as follows:

$$
\begin{aligned}
\eta & =\frac{P_{\text {output }}}{P_{\text {in }}}=\frac{T_{d}}{\left(\alpha T_{d} / \eta_{\mathrm{EM} 2}\right)+(1-\alpha) T_{d} / \eta_{\mathrm{EM} 1}} \\
& =\frac{1}{\alpha / f\left(n_{\mathrm{EM} 2}, \alpha T_{d}\right)+(1-\alpha) / f\left(n_{\mathrm{EM} 1},(1-\alpha) T_{d}\right)},
\end{aligned}
$$

where $\eta$ is the total efficiency of two motors, $P_{\text {output }}$ is total output power of two motors, and $P_{\text {in }}$ is the input power of the powertrain, which is supplied by the battery.

(2) Fundamental principle of adaptive nonlinear particle swarm optimization

Based on the powertrain model in Section 2 and the optimization problem description, it could be found that the optimization of the torque split ratio is a highly nonlinear optimization problem. The optimal torque split ratio can be described as the optimization of $\alpha$ within the simplified range, given the time-varying inputs $\left(T_{d}(k), n_{\mathrm{EM} 1}(k)\right.$, and $\left.n_{\mathrm{EM} 2}(k)\right)$, subject to a set of time-varying constraints $\left(T_{\text {dismaxEM1 }}(k), T_{\text {dismaxEM2 }}(k)\right)$.

PSO is a population-based heuristic algorithm introduced by Kennedy and Eberhart in 1995 [29], which mimics the social behavior of animal groups such as a flock of birds or fish shoals guiding particles to find global optimal solution. The optimization procedure can be abstracted as particles flying in the designed $N$-dimensional space. During each flying process, each particle would update its velocity and position according to previous individual best position and the best position of the swarm. Then, the particle will be compared with the best solution. It 
has experienced to update individual best position. And the best position of the swarm is further selected among all individual best positions. The velocity and position of each particle can be calculated using the following equation:

$$
\begin{aligned}
v_{i}^{N}(t+1)= & \xi v_{i}^{N}(t)+c_{1} r_{1}\left(\operatorname{pBest}_{i}^{N}(t)-p_{i}^{N}(t)\right) \\
& +c_{2} r_{2}\left(\operatorname{gBest}^{N}(t)-p_{i}^{N}(t)\right), \\
p_{i}^{N}(t+1)= & p_{i}^{N}(t)+v_{i}^{N}(t+1),
\end{aligned}
$$

where $v_{i}^{N}(t)$ is the current velocity of the $i^{\text {th }}$ particle, $i=1, \ldots$, sizepop; sizepop is the population size of the swarm; $t$ means the $t^{\text {th }}$ iteration, $t=1, \ldots$, maxgen; $N$ represents the dimensions of solution space; $\xi$ is the inertia weight, which is used to inherit from previous velocity feature to next iteration; $c_{1}$ and $c_{2}$ are the learning factors of individual learning and social learning, respectively; $r_{1}$ and $r_{2}$ are the random number between 0 and $1 ; \mathrm{pBest}_{i}^{N}(t)$ is the best position of the $i^{\text {th }}$ particle in $t^{\text {th }}$ iteration; $p_{i}^{N}(t)$ is the current position of the $i^{\text {th }}$ particle in $t^{\text {th }}$ iteration; and gBest ${ }^{N}(t)$ is the best position of the whole swarm after $t$ iterations.

It is proved that PSO is a simple, efficient, and robust stochastic optimization method to solve a nonlinear optimization problem using the concept of social interaction. However, the general PSO suffers from some drawbacks, which may result in premature convergence and slow convergence speed [30].

To improve the performance of general PSO, this paper adopts an adaptive nonlinear particle swarm optimization algorithm (ANLPSO). First, in the general PSO, the initial position and velocity of each particle are randomly generated within the designated range (range of the optimization variables). Assuming most of the initial particles centralized in a small region (subset of the designated range), the PSO would be highly possible to get into the local optimum, shown in Figure 8(a). As such, the initialization process is redesigned to guarantee the population diversity in the early optimization process. The designated range is divided into sizepop segments. The initial position of each particle is randomly generated within the range of corresponding segment, shown in Figure 8(b). Thus, the particle is distributed within the designated range as uniformly as possible, as shown in the following equation:

$$
p_{i}^{N}=p_{\min }^{N}+\frac{(\mathrm{i}-1)\left(p_{\max }^{N}-p_{\min }^{N}\right)}{\text { sizepop }}+\frac{r_{3}\left(p_{\max }^{N}-p_{\min }^{N}\right)}{\text { sizepop }}
$$

where $p_{\min }^{N}$ and $p_{\max }^{N}$ are lower bound and upper bound of the designated range, respectively, and $r_{3}$ is a random number between 0 and 1 .

Second, it is concluded that the bigger $\xi$ value results in the occurrence of exploration behavior, while the smaller $\xi$ value results in the occurrence of exploitation behavior [31]. In the ANLPSO, the inertia weight decreases nonlinearly with the increase in iteration. In the early optimization process, the inertia weight is relatively big to ensure the convergence speed. And the inertia weight decreases with the increasing iteration to improve the quality of solution. When compared to linear decreasing inertia weight, the inertia weight in ANLPSO is a little smaller, resulting in better quality of solution. The inertia weight can be determined as follows:

$$
\xi=\xi_{\max }+\left(\frac{t-1}{\operatorname{maxgen}-1}\right)^{0.5}\left(\xi_{\min }-\xi_{\max }\right),
$$

where $\xi_{\min }$ and $\xi_{\max }$ are the designated minimum value and maximum value of the inertia weight.

Third, the learning factors $c_{1}$ and $c_{2}$ are dynamically changing with the fitness value of current particle, shown in equation (19). Particles with above average fitness values can obtain the smaller $c_{1}$ value and bigger $c_{2}$ value to strengthen their social-learning abilities and improve solutions' qualities. On the contrary, particles with below average fitness values can obtain the bigger $c_{1}$ value and smaller $c_{2}$ value to strengthen their global search abilities and improve convergence speed:

$$
\begin{cases}c_{1}=c_{1, \max }-\frac{\left(c_{1, \max }-c_{1, \min }\right)\left(\mathrm{Fit}_{i}-\mathrm{Fit}_{\mathrm{min}}\right)}{\mathrm{Fit}_{\mathrm{avg}}-\mathrm{Fit}_{\mathrm{min}}}, & c_{2}=c-c_{1}, \mathrm{Fit}_{i}<\mathrm{Fit}_{\mathrm{avg}}, \\ c_{1}=c_{1, \min }, c_{2}=c-c_{1}, & \mathrm{Fit}_{i} \geq \mathrm{Fit}_{\mathrm{avg}},\end{cases}
$$

where $c_{1, \min }$ and $c_{1, \max }$ are minimum and maximum individual-learning factor value, respectively; $c$ is the designated sum value of $c_{1}$ and $c_{2}$; Fit ${ }_{i}$ is the fitness value of the $i^{\text {th }}$ particle; and Fit min $_{\text {and }}$ Fit $t_{\text {avg }}$ are the minimum and average fitness value of the whole swarm, respectively. 


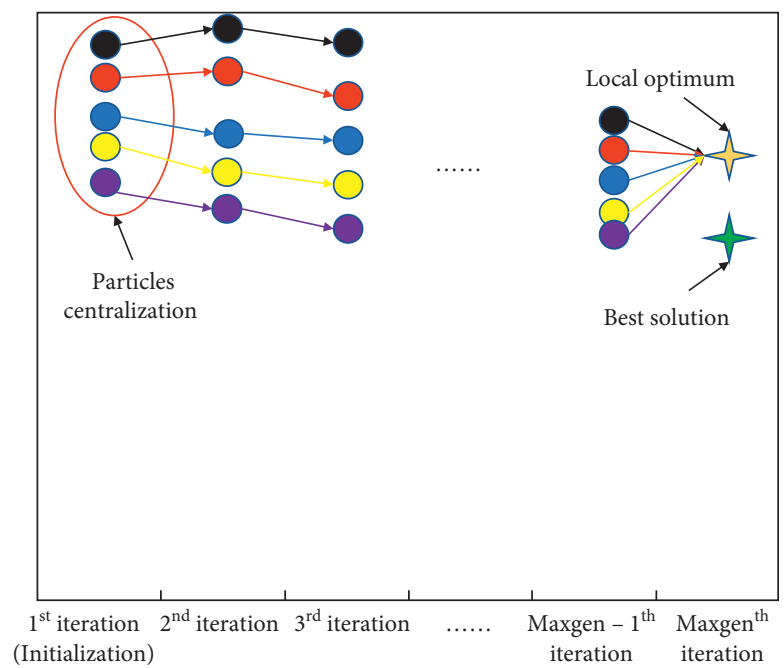

(a)

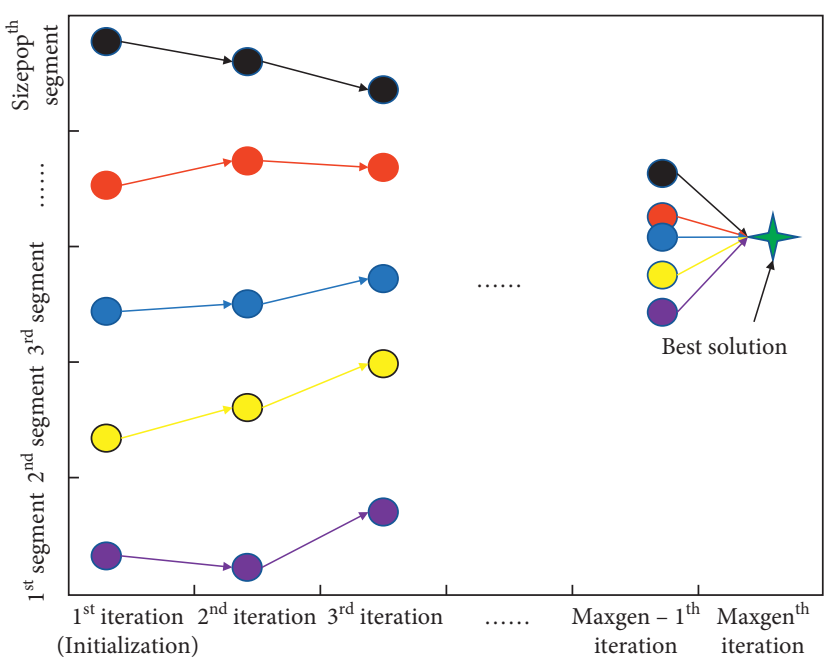

(b)

FIgURE 8: Differences in initialization process between (a) PSO and (b) ANLPSO.

(3) Procedures of adaptive nonlinear particle swarm optimization

To optimize the energy efficiency in this dual-motor vehicle, the ANLPSO algorithm was adopted to control the torque split between two motors. The optimization procedure of the torque split ratio based on ANLPSO (shown in Figure 9) is described as follows [32]:

(1) Adaptive initialization: the initial position of particles is generated according to equation (17) to guarantee the population diversity, while the initial velocity for the particles is randomly generated. The optimization problem in this study is a one-dimensional $(N=1)$ problem. Hence, the position and velocity of the particles are described as $\alpha_{i}$ and $v_{i}$, respectively, which are generated as follows:

$$
\begin{aligned}
& \alpha_{i}=\alpha_{\min }+\frac{(i-1)\left(\alpha_{\max }-\alpha_{\min }\right)}{\text { sizepop }}+\frac{r_{3}\left(\alpha_{\max }-\alpha_{\min }\right)}{\text { sizepop }}, \\
& v_{i}=R\left[v_{\min }, v_{\max }\right],
\end{aligned}
$$

where $\alpha_{\min }$ and $\alpha_{\max }$ are lower and upper boundaries of torque split ratio, respectively, and $R\left[v_{\min }, v_{\max }\right]$ means the random process to generate velocity within the range of minimum velocity and maximum velocity.

(2) Update inertia weight and learning factors: the nonlinear-decreasing inertia weight and dynamic learning factors can be determined according to equations (18) and (19), respectively.

(3) Update velocity and position: the velocity and position of each particle are updated according to equation (16).
(4) Fitness value calculation: as mentioned, the total efficiency is used as objective function of the optimization problem. In ANLPSO, the fitness value of the particle is calculated based on this objective function. And the particle with the maximum fitness value would be selected as the best solution:

$$
\text { Fit }=\eta=\frac{1}{\alpha / f\left(n_{\mathrm{EM} 2}, \alpha T_{d}\right)+(1-\alpha) / f\left(n_{\mathrm{EM} 1},(1-\alpha) T_{d}\right)} \text {. }
$$

(5) Update pBest ${ }_{i}$ and gBest: after the calculation of fitness value, each particle should choose the particle with the maximum fitness value, pBest $_{i}$, according to its own experience. Besides, the particle with global best fitness value of the whole swarm should be selected as gBest among all pBest ${ }_{i}$. In the first iteration, each particle is directly selected as its own pBest ${ }_{i}$.

(6) Stopping rule: Steps (2)-(5) are repeated until the optimization converges or the maximum number of iteration is reached. The best position with the maximum fitness value would be outputted as the optimal torque split ratio of two motors.

\section{Results and Discussion}

In this study, the simulation was conducted for four cases, including single-motor powertrain, dual-motor powertrain with even distributed strategy, dual-motor powertrain with rule-based strategy, and dual-motor powertrain with ANLPSO strategy. The results would be analyzed with comparison in this section. It is noted that the torque split strategy was not applied into the control strategy for single-motor powertrain. Thus, only the results of energy consumption would be compared with other three cases. 


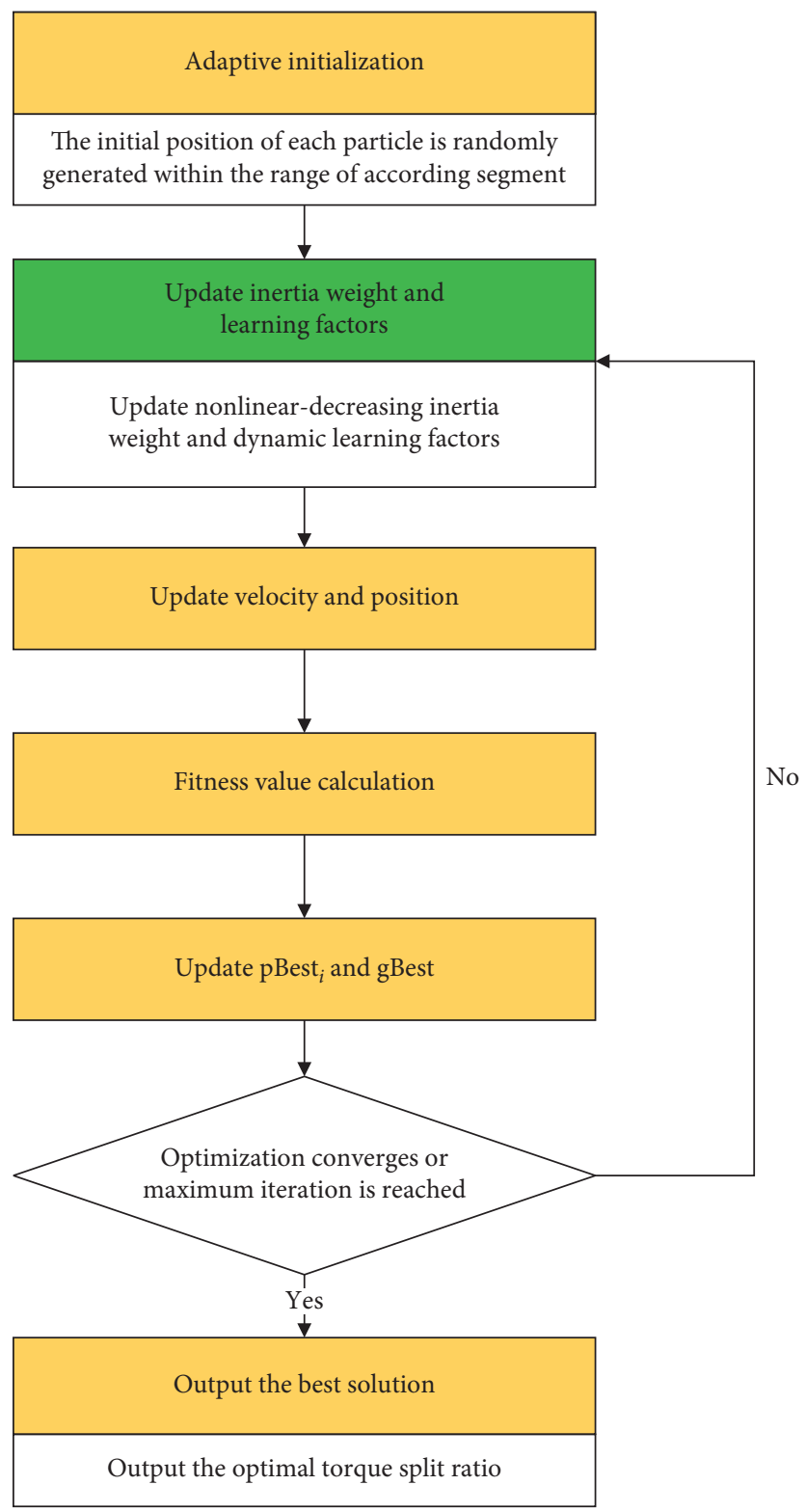

FIgURE 9: Optimization procedure of ANLPSO.

4.1. Simulation Program and Settings. The simulation was mainly based on Matlab/Simulink platform. The sampling time was set as a fixed sampling time of $0.1 \mathrm{~s}$, and the Bogacki-Shampine solver was selected. Both the driver model, physical vehicle model, and vehicle control unit (VCU) model were conducted, as shown in Figure 10. The ANLPSO strategy was programmed as a S-function block in the torque split layer of VCU. All key parameters of ANLPSO are listed in Table 4.

4.2. Simulation Model Validation. In order to verify the validity and accuracy of the physical simulation model, the real vehicle experiment is conducted. As mentioned in Section 2, the target vehicle is a commercial dual-motor electric vehicle prototype. The powertrain architecture of real vehicle is shown in Figure 11(a). In the development stage, the allocation of driving torque for this sample vehicle is based on the simplest even distributed strategy and the braking energy is not regenerated. Due to the restricted experiment condition, the real-vehicle experiment was conducted on chassis dynamometer using the NEDC (as shown in Figure 11(b)). Both the vehicle velocity and battery SOC results were recorded from VCU through CAN bus acquisition tool and used to validate the corresponding simulation model. It is noted that all parameters and the control strategy of the simulation model are consistent with the experiment.

As shown in Figure 12(a), both the simulation model and experimental vehicle could track the driving cycle well. The vast majority of velocity tracking error of experiment results is within $\pm 5 \mathrm{~km} / \mathrm{h}$, while the velocity tracking error of 


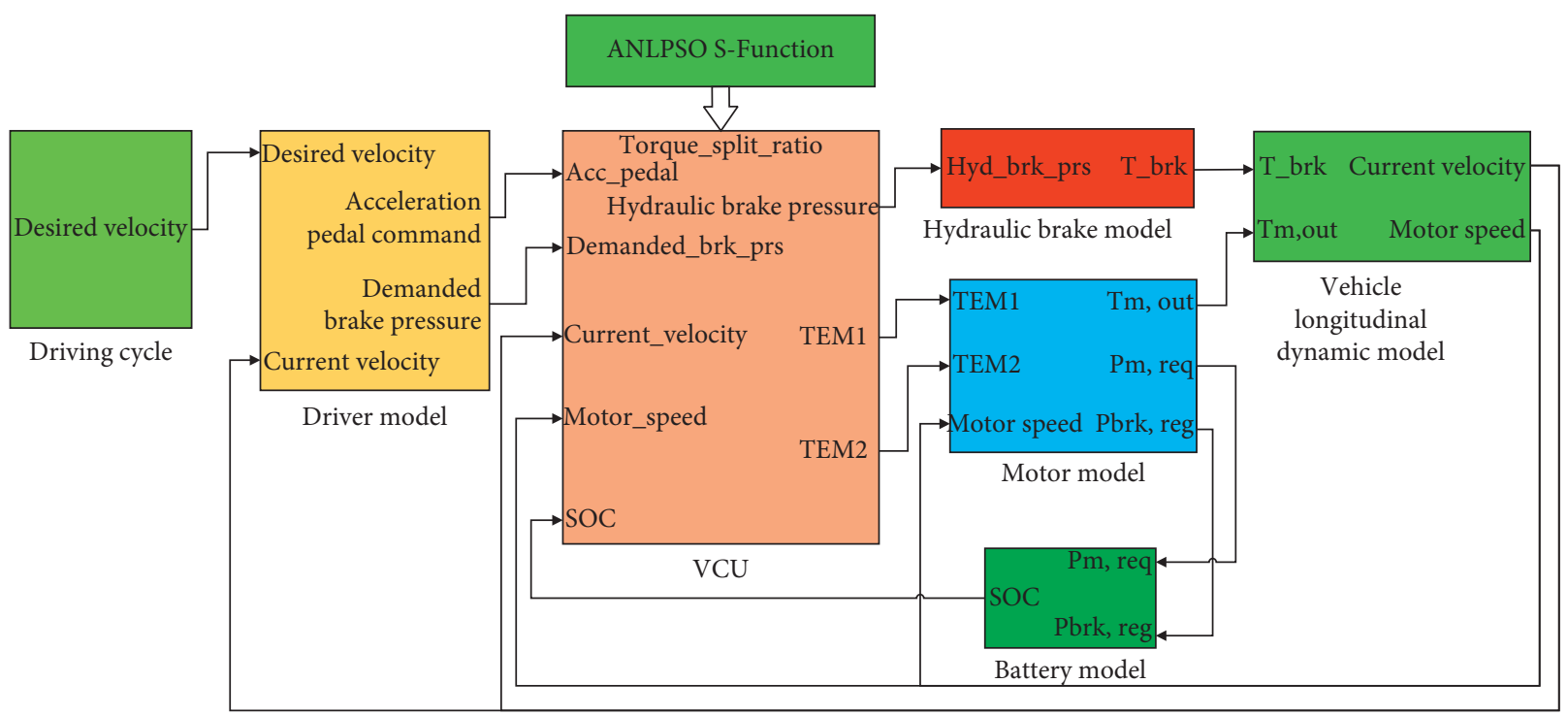

Figure 10: Physical vehicle model with VCU based on Matlab/Simulink platform.

TABLE 4: Parameters value of ANLPSO.

\begin{tabular}{lc}
\hline Parameters & Value \\
\hline Sizepop & 5 \\
Maxgen & 20 \\
$N$ & 1 \\
$\alpha_{\min }$ & 0 \\
$\alpha_{\max }$ & 0.5 \\
$\xi_{\min }$ & 0.4 \\
$\xi_{\max }$ & 0.9 \\
$v_{\min }$ & -0.25 \\
$v_{\max }$ & 0.25 \\
$c_{1, \min }$ & 1.2 \\
$c_{1, \max }$ & 1.5 \\
$c$ & 3 \\
\hline
\end{tabular}

simulation is within $\pm 0.5 \mathrm{~km} / \mathrm{h}$. As for the changing tendency of battery SOC, simulation and experimental results are consistent. Because of the sampling accuracy $(0.4 \%)$ of battery management system, the SOC results of experiment decrease in a ladder shape. The SOC starts with an initial value of $90 \%$ and ends with $84.87 \%$ and $84.8 \%$ in simulation and experiment, respectively, implying a high validity and accuracy of the simulation model, shown in Figure 12(b).

4.3. Simulation Results. With the validated simulation model, comparative simulation results are presented using three different strategies (note that regenerative braking strategy is added to controller in this section). The results in NEDC at the initial SOC 90\% are demonstrated in Figures 13 and 14. As illustrated in the first row of Figure 13, the actual velocity $\left(V_{-} c\right)$ tracks the desired velocity $\left(V_{-} d\right)$ very well for all three cases. The velocity track error is within $\pm 0.4 \mathrm{~km} / \mathrm{h}$ since more precise driver model based on the feedforwardfeedback PI control is adopted, indicating the reliability of the simulation results. The second row presents the torque split ratio $(\alpha)$ results for even distributed strategy. It is

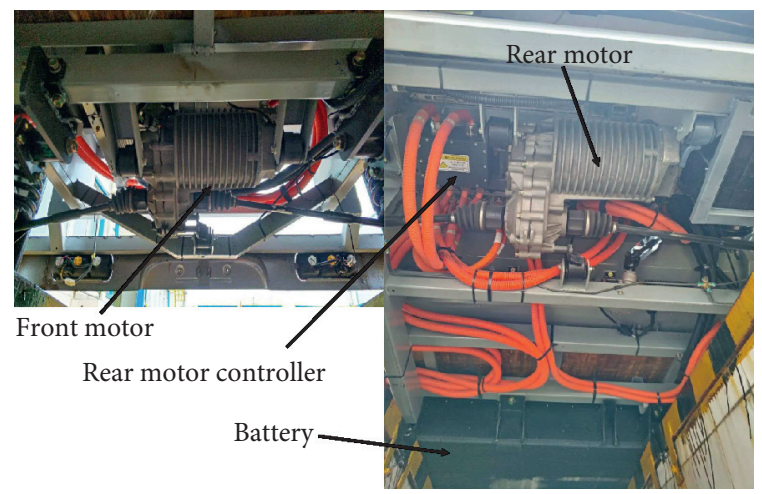

(a)

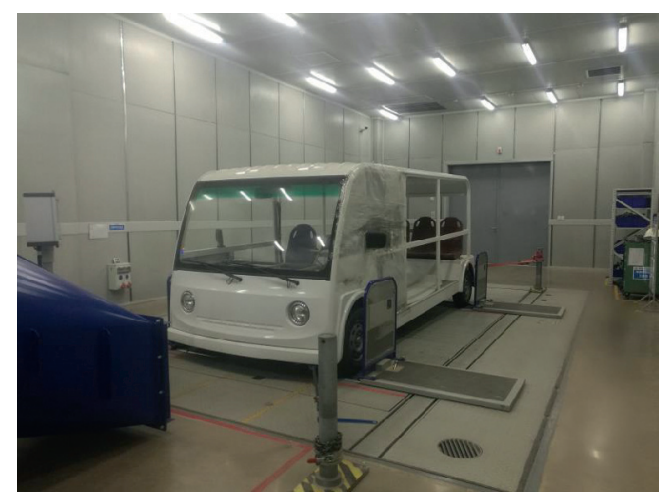

(b)

FIGURE 11: Real-vehicle experiment: (a) powertrain architecture; (b) vehicle experiment on dynamometer.

obvious that the torque split ratio is 0.5 during acceleration and uniform velocity conditions (T_r $>0$ ), which means the desired driving torque is evenly distributed between EM1 and EM2. However, the torque split ratio is 0 in the third row for rule-based strategy, indicating that the EM1 provides driving torque solely in the whole cycle run. In the fourth 


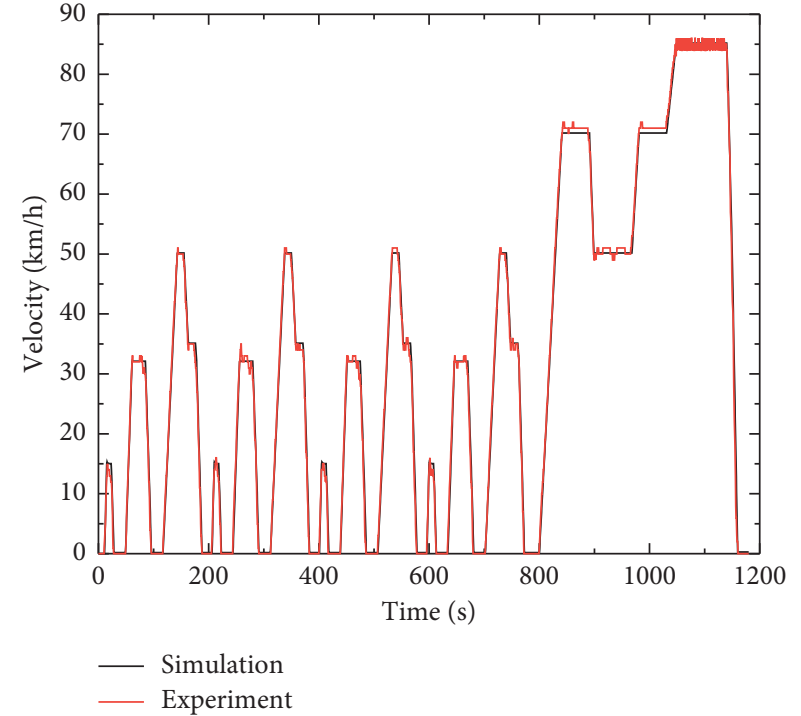

(a)

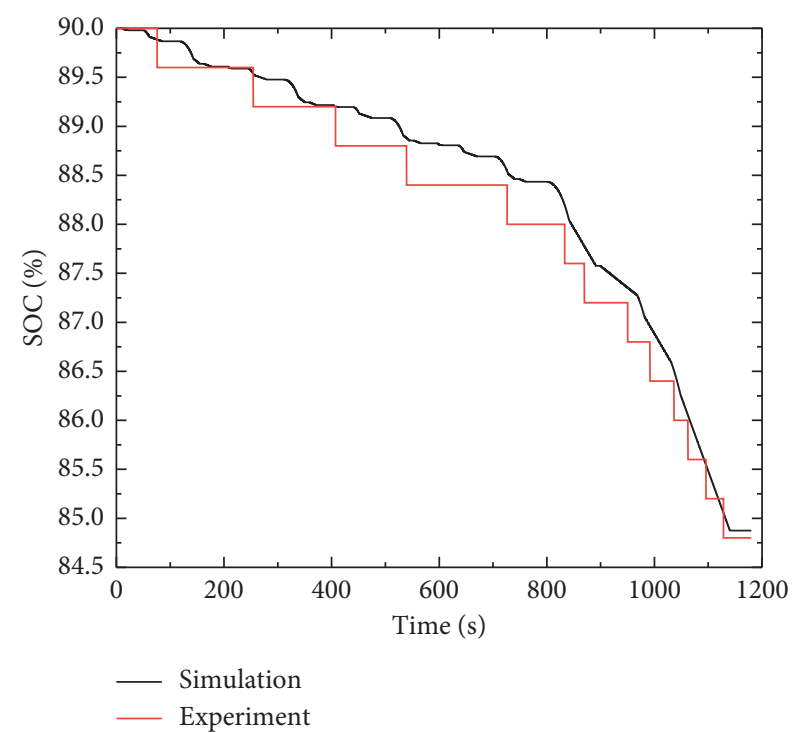

(b)

FIgURE 12: Comparison of simulation and experiment results: (a) driving cycle tracking; (b) battery SOC.
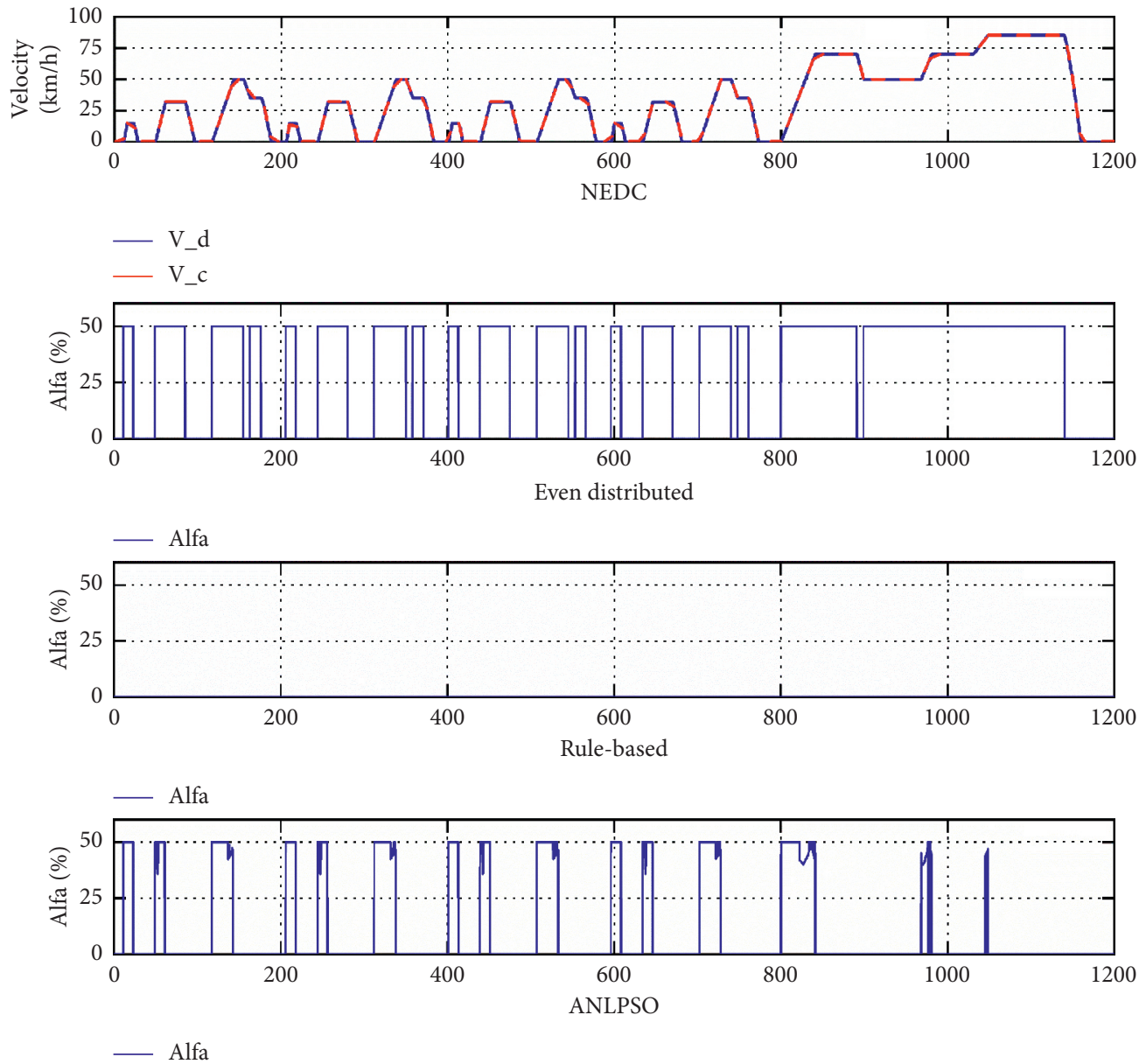

Figure 13: Velocity and driving torque split ratio of three strategies in NEDC. 


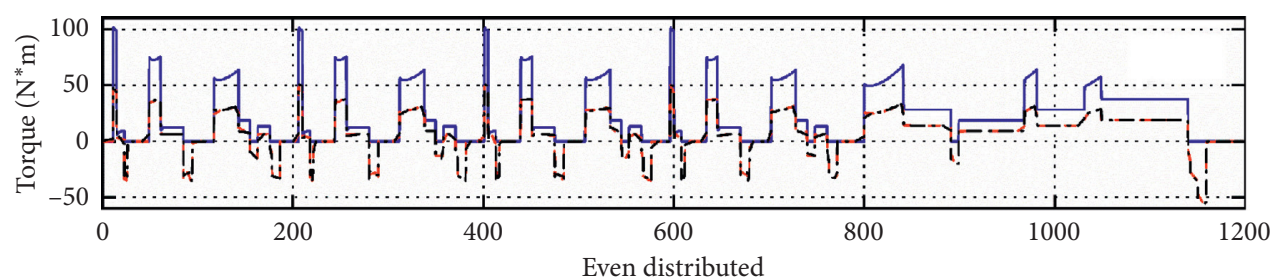

- T_r

- - - T_m1

...- T_m2

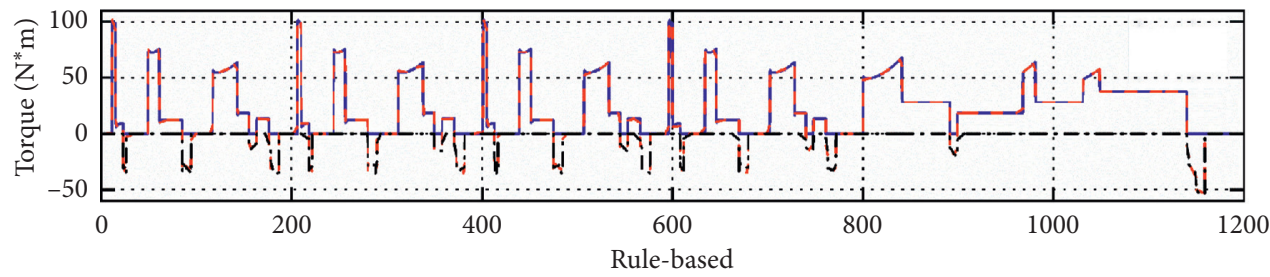

- T_r

--- T_m1

-..- T_m2

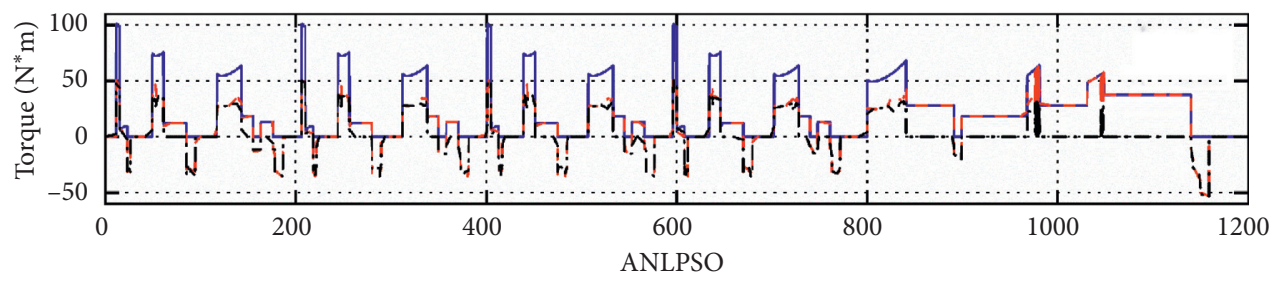

- T_r

-.- T_m1

...- T_m2

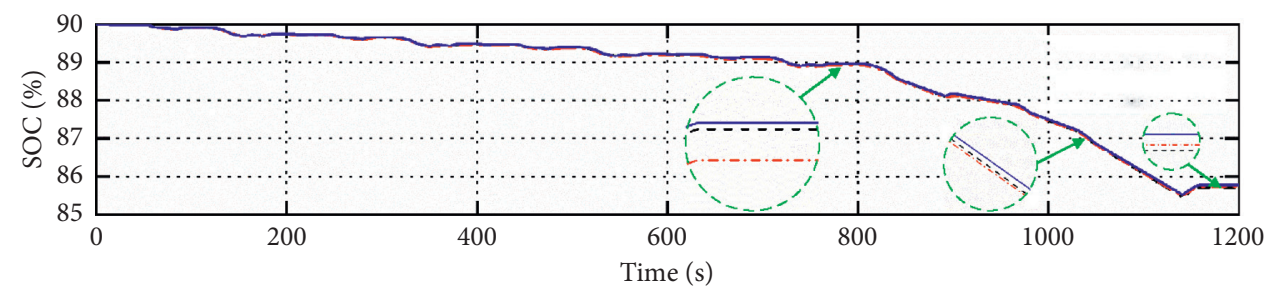

Even distributed

- - - Rule-based

... ANLPSO

FIgURE 14: Torque curves and the battery SOC of three strategies in NEDC.

row, the real-time torque split ratio results based on ANLPSO is revealed. Compared with the other two strategies, the optimal torque split ratio changes with torque requirements during acceleration condition, which implies that two motors participate in propulsion more flexibly to achieve a higher total efficiency.

In order to elucidate the difference of those three strategies specifically, the torque results of three cases are illustrated in Figure 14. For the even distributed strategy in the first row, the demanded torque is equally distributed to EM1 and EM2. The torque change trajectory of two motors is exactly the same. As for rule-based strategy, the vehicle is propelled only by the EM1, and the torque change curve of EM1 is the same as that of demanded torque. The torque results are in concert with the torque split ratio results shown in Figure 13. With respect to ANLPSO, the torque of two motors is split based on $\alpha$. The EM1 predominates in the low torque area (when T_r is small, such as uniform velocity condition), while two motors are both activated to propel the vehicle in high torque area (when T_r is relatively big, such as acceleration condition). Within the high torque area, the torque split ratio is 0.5 mostly. However, it is noticeable that the required torque is not equally distributed to two motors in some of high torque area, which indicates that even distributed strategy is not always the optimal torque split 

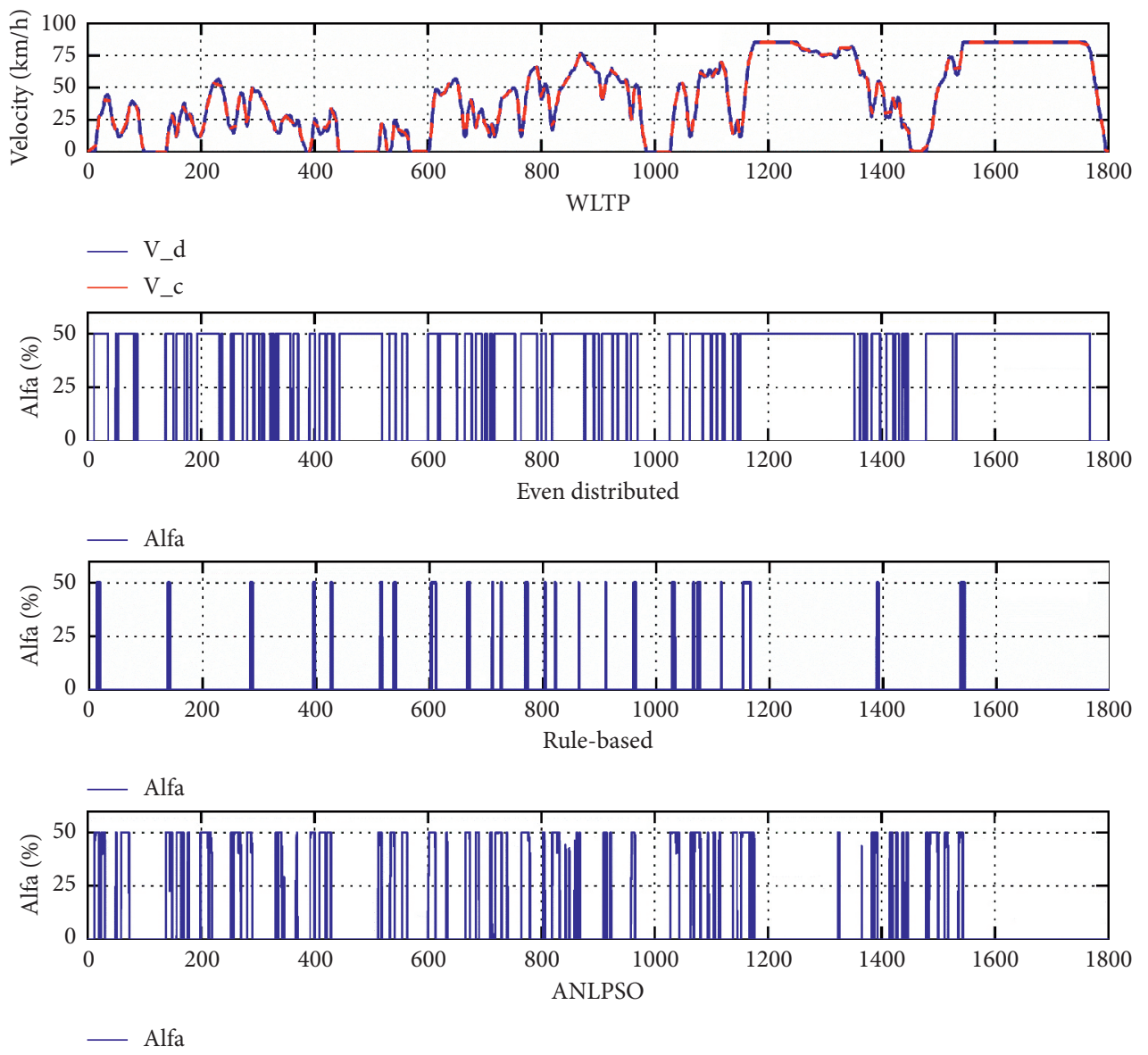

FIGURE 15: Velocity and driving torque split ratio of three strategies in WLTP.

strategy in high required torque area (in literature review, for dual-motor powertrain with two identical motors, the optimal torque split strategy is even distributed strategy, while the high driving torque is required). The fourth row shows SOC change trajectory of three cases. Since the powertrain is the same, the difference between three cases is small. As can be seen, there are three green dotted circles, which present three zoom-in pictures of SOC change curve in low velocity area, medium velocity area, and high velocity area, respectively. The battery SOC of ANLPSO is always higher than the rest two cases, indicating that the torque split strategy with ANLPSO consumes least energy. The rule-based strategy is least efficient because the required torque is relatively high in most time of first $800 \mathrm{~s}$ as it is inefficient that single motor drives the vehicle in high torque area. After 800 s, most time of cycle run is in the area of uniform velocity, which means the required torque is low. It turns out that single motor is more efficient to supply the power. Furthermore, it can be seen that the difference of battery SOC between even distributed strategy and rule-based strategy becomes smaller and the SOC with rule-based strategy is higher than that with even distributed strategy eventually.

Figures 15 and 16present the simulation results in WLTP. As illustrated in Figure 15, the adaptive driver model still tracks the desired velocity precisely. It is apparent that acceleration and deceleration condition is more compared with NEDC. Consequently, EM2 works more frequently to assist EM1 to propel the vehicle in rule-based strategy, and the optimal torque split ratio based on ANLPSO also changes more frequently to achieve the best energy efficiency. Figure 16 demonstrates the comparison of torque results of three strategies. Similar with the results shown in Figure 14, it is more efficient that single motor supplies the power solely in low torque area, while two motors propel the vehicle together with an appropriate torque split ratio in high torque area. In the fourth row of Figure 16, the results of battery SOC is presented. It is obvious that more electricity is consumed in WLTP relative to in NEDC. In the first 1000s, frequent rapid acceleration indicates a higher required torque. Hence, the battery SOC of rule-based strategy drops more than that of even distributed strategy. Since the best split ratio is not always 0.5 in high torque area, energy efficiency of ANLPSO is still higher than that of even distributed strategy.

After $1100 \mathrm{~s}$, rule-based strategy consumes little energy than even distributed strategy because of the smoother acceleration process and uniform velocity period. And the change tendency of the SOC is similar to the results in the fourth row of Figure 14. In general, ANLPSO exhibits highest energy efficiency while the rule-based strategy is relatively efficient than even distributed strategy in both NEDC and WLTP. 


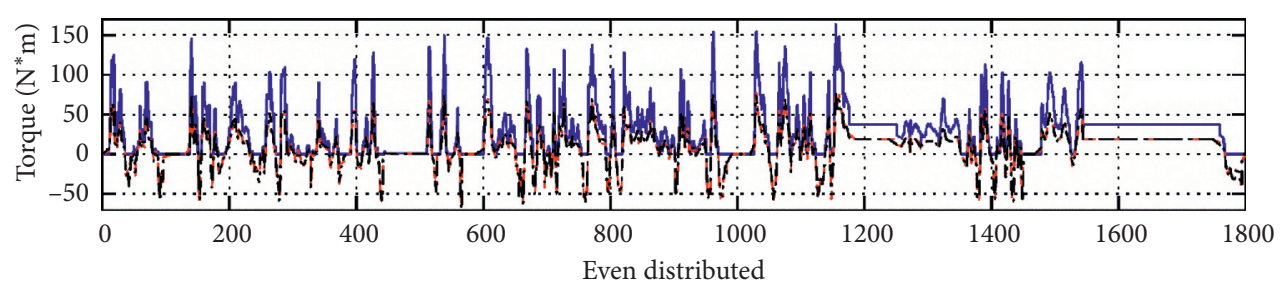

- T_r

-.- T_ml

...- T_m2

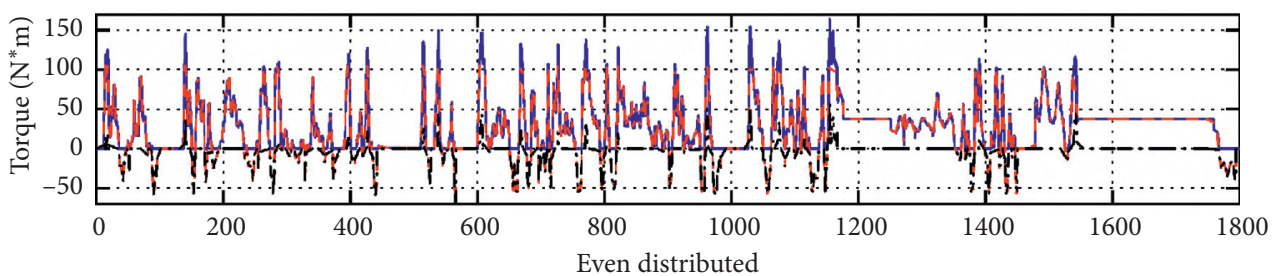

- T_r

-.- T_m1

...- T_m2

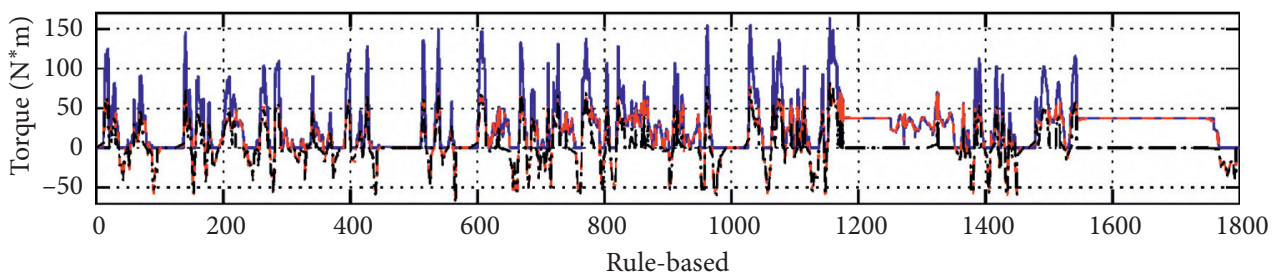

- T_r

-.- T_m1

-... T_m2

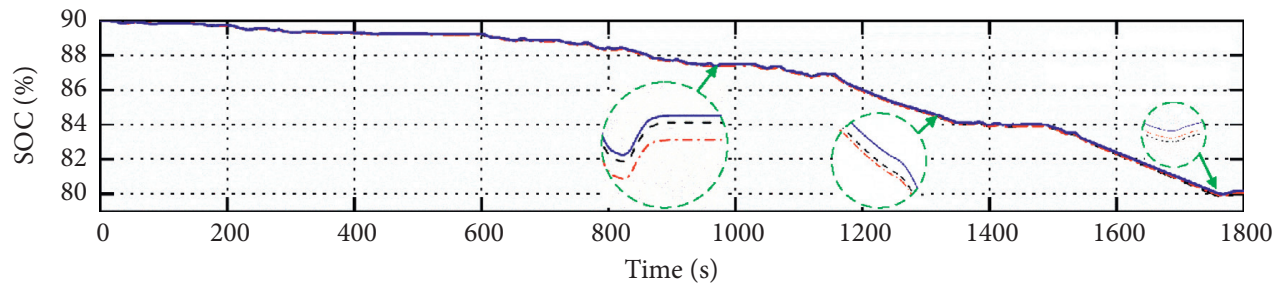

$$
\begin{aligned}
& \text { _ Even distributed } \\
& \text { - - } \text { Rule-based } \\
& \text {.. - ANLPSO }
\end{aligned}
$$

FIgURE 16: Torque curves and the battery SOC of three strategies in WLTP.

4.4. Optimization Performance and Real-Time Applicability Analysis of ANLPSO. As detailed in Section 3, the torque split ratio is searched based on ANLPSO, which is designed to ameliorate shortcomings of general PSO. To further compare the optimization performance and real-time applicability between general PSO and ANLPSO, the evolution process was investigated. Figure 17 shows the optimization process to search the best torque split ratio at T_r $=85 \mathrm{Nm}$ and Motor_speed $=5400 \mathrm{rpm}$ (to compare two methods fairly, this condition is randomly selected from cycle run). Both the best position of each particle $P_{\text {best }}$, the best position of all particles $G_{\text {best }}$, and the best fitness value in each iteration are demonstrated. As shown in the first row, four particles are randomly generated within a small range (0.35-0.36). As a result, the optimization process is trapped in local optimum at the $7^{\text {th }}$ iteration, and thus, the optimal solution is not found eventually. By contrast, because of the improvement of the initialization process, the nonlinear decreasing inertia weight, and dynamic learning factors, the converge speed and optimization performance of ANLPSO are greatly enhanced. As shown in the second row and the third row, the evolution process is more stable and swifter. The optimal fitness value (fitness value $=93.77345$ ) is found only after 9 iterations, and the best position of each particle $P_{\text {best }}$ both converges to the optimal position $(\alpha=0.449)$ after 16 iterations. Therefore, ANLPSO is verified to possess better 


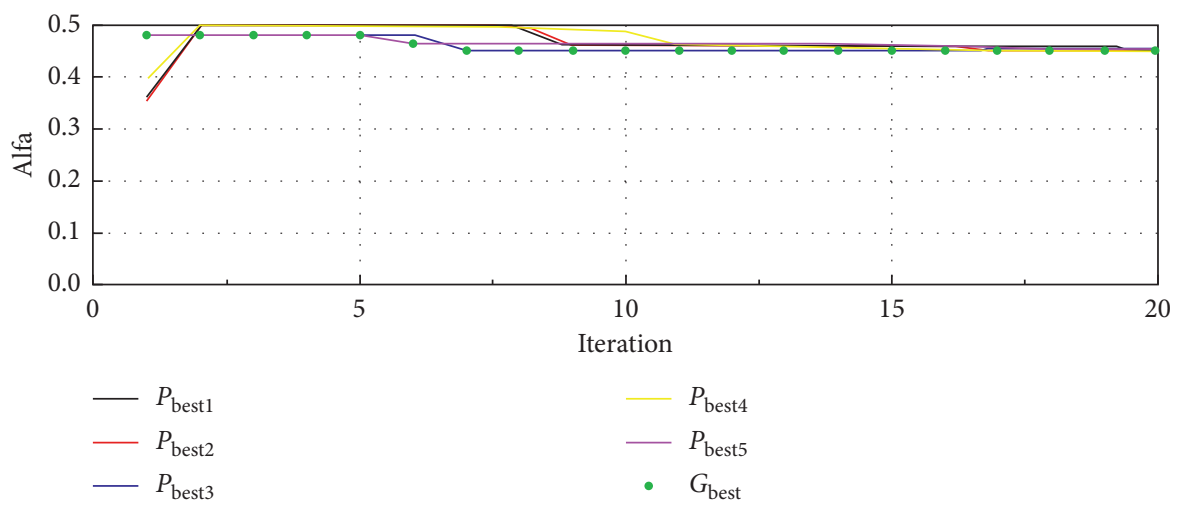

(a)

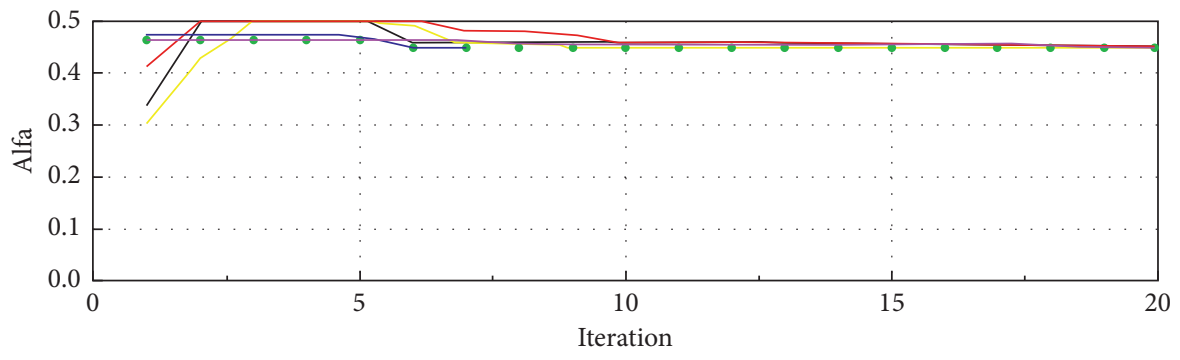
$-P_{\text {bestl }}$
- $P_{\text {best2 }}$
- $P_{\text {best3 }}$
$P_{\text {best4 }}$
- $P_{\text {best5 }}$
- $G_{\text {best }}$

(b)

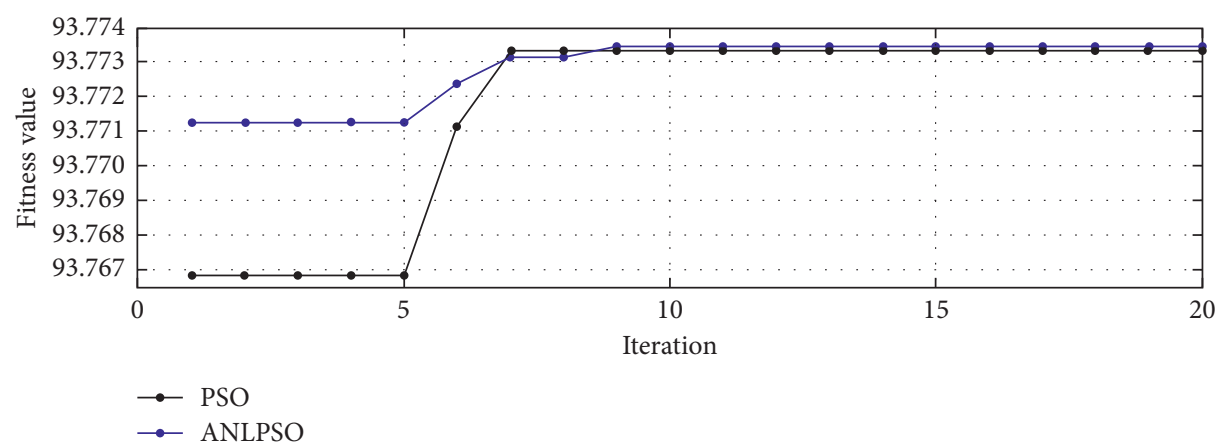

(c)

Figure 17: Comparison of convergence performance between PSO and ANLPSO.

convergence performance and global optimization ability than general PSO.

Furthermore, the computational time of the optimal torque split strategies is $377 \mathrm{~s}$ and $665 \mathrm{~s}$ for NEDC (1180 s) and WLTP $(1800 \mathrm{~s})$, respectively. Then, the equivalent computational time per second is approximately $0.031 \mathrm{~s}$ and $0.036 \mathrm{~s}$, which shows a great real-time applicability and potential to be implemented in VCU of real vehicle. Before practical applications, to decide the population size and maximum number of iteration, there should be a tradeoff between the optimality and real-time applicability. For example, as for VCU with limited computational capability, it is probably advisable to use a relatively small maximum iteration size and a relatively small population size (10 iterations and 5 populations) to ensure real-time applicability. By contrast, it would be better to determine a large maximum iteration size and a large population size (30 iterations and 10 populations) to take priority of the optimality without sacrifice in real-time implementability.

4.5. Comparison of Efficiency, Motor Loss, and Energy Improvement. To further demonstrate energy-saving potential of dual-motor powertrain over single-motor powertrain and the difference of three control strategies, the total efficiency of powertrain, the power loss of two motors, and energy consumption were investigated. Figure 18 illustrates the results of total efficiency of two motors in NEDC and 


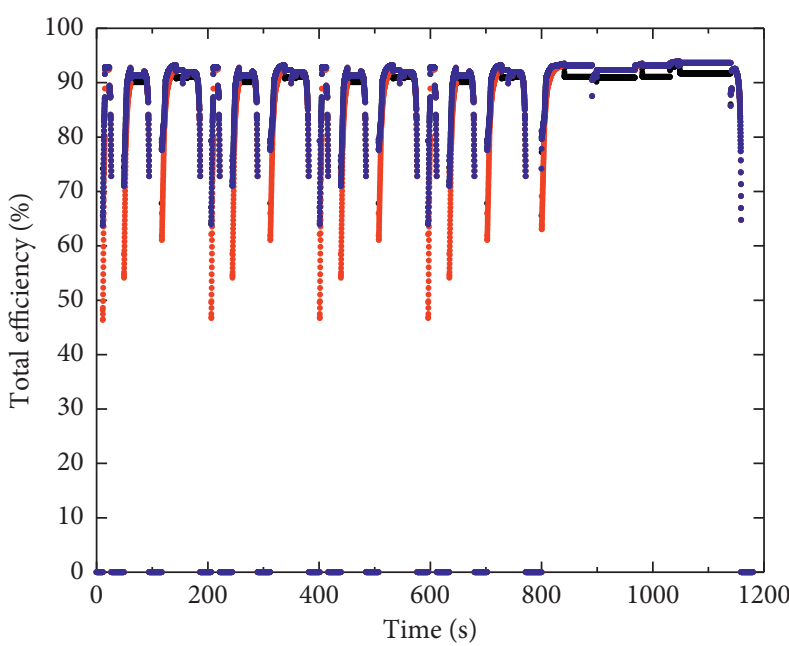

- Even distributed

- Rule-based

- ANLPSO

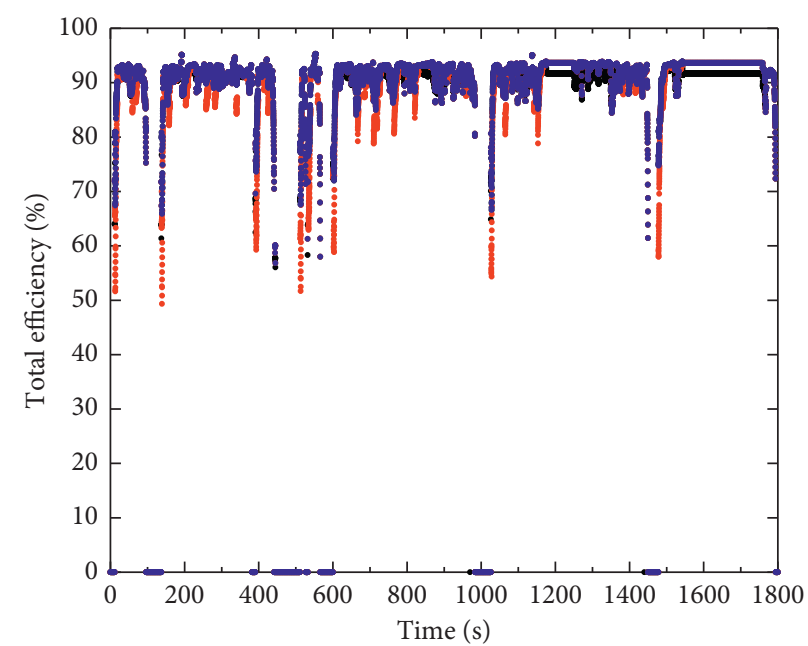

- Even distributed

- Rule-based

- ANLPSO

(a)

(b)

FIgURE 18: Total efficiency of two motors of three strategies in NEDC and WLTP.

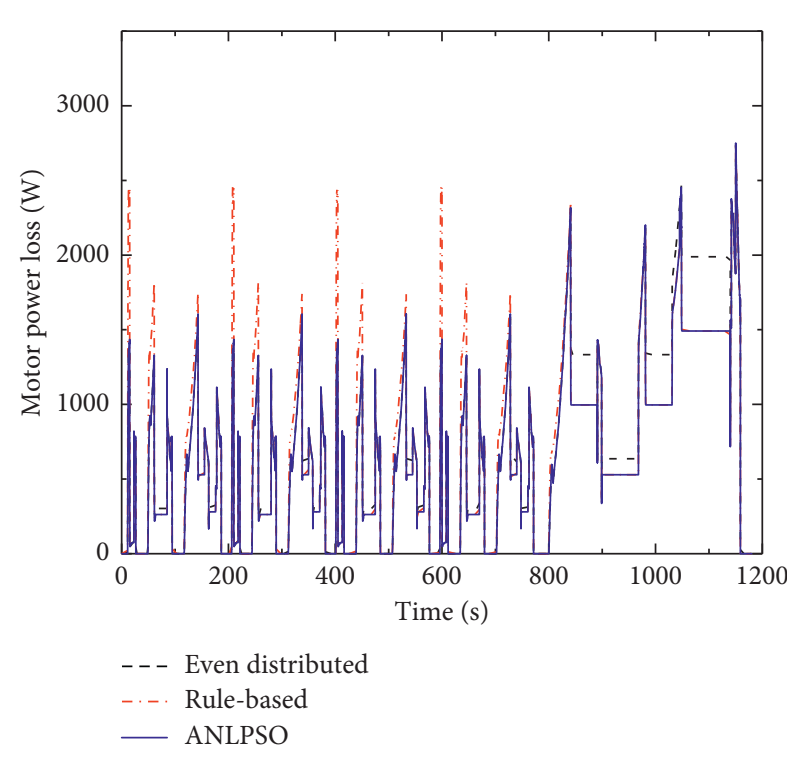

(a)

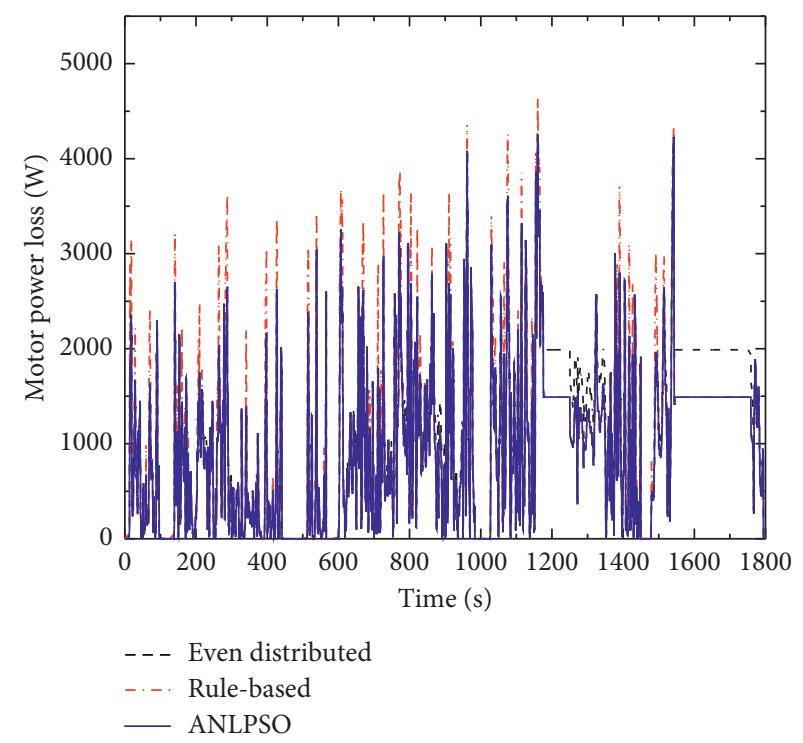

(b)

FIGURE 19: Motor power loss of two motors of three strategies in NEDC and WLTP.

WLTP. The total efficiency of ANLPSO is above $90 \%$ in most of cycle run, and even the lowest efficiency is not lower than $65 \%$, which is the highest among three strategies. Consequently, the power loss of two motors is the lowest with ANLPSO, as shown in Figure 19.

As for rule-based strategy, the total efficiency is the lowest in the first $800 \mathrm{~s}$ in NEDC and in the first $1000 \mathrm{~s}$ in WLTP. However, it becomes more efficient than even distributed strategy when the cycle run enters in low torque area (after $800 \mathrm{~s}$ in NEDC and after $1000 \mathrm{~s}$ in WLTP). And it is obvious that the motor power loss of rule-based strategy is the most in high torque area and the motor power loss of even distributed strategy is the most in low torque area, as shown in Figure 19. It is worth noting that the total efficiency of even distributed strategy is basically the same as ANLPSO in high torque area, while the total efficiency of rule-based strategy is the same as ANLPSO in low torque area.

The energy consumption is obtained by integrating the battery power in NEDC and WLTP, as listed in Table 5. Assuming that the braking energy of each axle is totally regenerated by the motor mounted on according axle, it is 
TABLE 5: Energy consumption comparison.

\begin{tabular}{|c|c|c|c|c|c|c|}
\hline & \multicolumn{2}{|c|}{$\begin{array}{l}\text { Total motor loss } \\
(\mathrm{kWh})\end{array}$} & \multicolumn{2}{|c|}{$\begin{array}{c}\text { Electricity } \\
\text { consumption } \\
(\mathrm{kWh} / 100 \mathrm{~km})\end{array}$} & \multicolumn{2}{|c|}{$\begin{array}{c}\text { Energy saving } \\
\text { compared to single- } \\
\text { motor powertrain } \\
(\%)\end{array}$} \\
\hline & NEDC & WLTP & NEDC & WLTP & NEDC & WLTP \\
\hline Single-motor powertrain & - & - & 20.29 & 23.16 & - & - \\
\hline Dual-motor powertrain with even distributed strategy & 0.2324 & 0.5330 & 18.15 & 20.65 & 10.55 & 10.83 \\
\hline Dual-motor powertrain with rule-based strategy & 0.2221 & 0.5107 & 18.05 & 20.54 & 11.04 & 11.31 \\
\hline Dual-motor powertrain with ANLPSO & 0.2041 & 0.4695 & 17.88 & 20.34 & 11.88 & 12.18 \\
\hline
\end{tabular}

proved that the energy saving of dual-motor powertrain with three different strategies in NEDC has been improved by around $10.55 \%, 11.04 \%$, and $11.88 \%$, respectively, while the energy saving improvement of dual-motor powertrain with three different strategies in WLTP is $10.83 \%, 11.31 \%$, and $12.18 \%$, respectively, compared with single-motor powertrain. In addition, when compared with even distributed strategy and rule-based strategy for dual-motor powertrain, the proposed torque split strategy shows an improvement in total motor loss of $12.17 \%$ and $8.1 \%$ in NEDC, respectively, and $11.91 \%$ and $8.07 \%$, respectively, in WLTP.

\section{Conclusion}

In this study, a real-time optimal energy management strategy for a dual-motor electric powertrain was proposed. Firstly, the physical vehicle model was implemented in Matlab/Simulink platform. Then, the optimal torque split strategy based on ANLPSO was integrated with VCU by using S-function. Moreover, three cases (single-motor powertrain, dual-motor powertrain with even distributed strategy, and dual-motor powertrain with rule-based strategy) were also modeled for comparison. Several important findings are summarized as follows:

(1) In NEDC and WLTP driving cycles, the dual-motor powertrain with two identical motors could achieve energy saving of $(10.55 \%, 10.83 \%),(11.04 \%, 11.34 \%)$, and $(11.88 \%, 12.18 \%)$ using even distributed strategy, rule-based strategy, and the optimal torque split strategy, compared to single-motor powertrain, respectively. This confirms that the dual-motor powertrain has a great energy efficiency improvement over single-motor powertrain.

(2) With respect to dual-motor powertrain, compared to even distributed strategy, the proposed optimal torque split strategy could reduce the total motor loss by $12.17 \%$ and $11.91 \%$ in NEDC and WLTP, respectively. And the improvement over rule-based strategy is $8.1 \%$ and $8.07 \%$ accordingly.

(3) As for the ANLPSO, the real-time best torque split ratio could be searched swiftly with three inputs (motor speed, the required torque, and the available maximum torque of each motor) and fitness function of total efficiency of two motors. It is also shown that it has better convergence speed and global optimization performance than conventional PSO, which is provably real-time available in a real controller to achieve the optimal torque allocation.

(4) Based on the detailed result analysis of three strategies, it is concluded that, for dual-motor with two identical motors, the single motor is more efficient to provide the power in low torque area. However, within high torque area, both two motors should be activated to propel the vehicle with an appropriate torque split ratio. In most cases, the optimal torque split ratio is 0.5 , which means the torque should be equally distributed to two motors. However, 0.5 is not always the best split ratio according to searching results of ANLPSO. Hence, a proper methodology to figure out the real-time optimal torque split ratio is essential to manage the torque distribution of dual motor powertrain, which is also the main contribution of this study.

Our future work could focus on the applicability of the optimal torque split strategy in the real vehicle test. Furthermore, the research would be expanded into the integrated optimization framework for component sizing and optimal energy management strategy for dual-motor powertrain.

\section{Data Availability}

The following data, models, or results that support the findings of this study are available from the corresponding author upon reasonable request: (1) vehicle parameters; (2) motor efficiency data; (3) vehicle physical model; and (4) results data.

\section{Conflicts of Interest}

The authors declare that there are no conflicts of interest.

\section{Acknowledgments}

The authors would like to express their gratitude to Tsinghua University under project 2017YFB0103902, Wuhan University of Technology under the 111 project (no. B17034), and Innovative Research Team Development Program of Ministry of Education of China (no. IRT_17R83) for supporting this study. 


\section{Supplementary Materials}

In the "experiment results" folder, the excel file named "experiment results" is the results from real-vehicle experiment validation, while the excel file named "simulation model" is the results of the validated simulation model. These results are mainly for simulation model validation. In the "simulation results" folder, there are two folders named "NEDC" and "WLTP," respectively, which present the simulation results in NEDC and WLTP, respectively. The excel file named "EVEN" is the results of even distributed strategy. The excel file named "Rule-based" is the results of the rule-based strategy. The excel file named "ANLPSO" is the results of the optimal torque split strategy using ANLPSO. These results are mainly for comparison between different torque split strategies. (Supplementary Materials)

\section{References}

[1] L. C. A. Silva, J. J. Eckert, F. M. Santiciolli, E. S. Costa, F. G. Dedini, and F. C. Correa, "A study of battery power for a different electric vehicle propulsion system," in Proceedings of the 2015 International Conference on Electrical Systems for Aircraft, Railway, Ship Propulsion and Road Vehicles (ESARS), pp. 1-6, IEEE, Aachen, Germany, March 2015.

[2] T. Wilberforce, Z. El-Hassan, F. N. Khatib et al., "Developments of electric cars and fuel cell hydrogen electric cars," International Journal of Hydrogen Energy, vol. 42, no. 40, pp. 25695-25734, 2017.

[3] K. T. Chau and C. C. Chan, "Emerging energy-efficient technologies for hybrid electric vehicles," Proceedings of the IEEE, vol. 95, no. 4, pp. 821-835, 2007.

[4] G. Wu, X. Zhang, and Z. Dong, "Powertrain architectures of electrified vehicles: review, classification and comparison," Journal of the Franklin Institute, vol. 352, no. 2, pp. 425-448, 2015.

[5] H. Eckard and P. Marx, "Electric cars: technical characteristics and environmental impacts," Environmental Sciences Europe, vol. 24, no. 1, pp. 1-15, 2012.

[6] O. van Vliet, A. S. Brouwer, T. Kuramochi, M. van den Broek, and A. Faaij, "Energy use, cost and $\mathrm{CO}_{2}$ emissions of electric cars," Journal of Power Sources, vol. 196, no. 4, pp. 2298-2310, 2011.

[7] S. Chopra and P. Bauer, "Driving range extension of EV with on-road contactless power transfer: a case study," IEEE Transactions on Industrial Electronics, vol. 60, no. 1, pp. 329-338, 2013.

[8] M. A. Miller, A. G. Holmes, B. M. Conlon, and P. J. Savagian, "The GM "voltec" 4ET50 multi-mode electric transaxle," SAE International Journal of Engines, vol. 4, no. 1, pp. 1102-1114, 2011.

[9] F. Di Nicola, A. Sorniotti, T. Holdstock, F. Viotto, and S. Bertolotto, "Optimization of a multiple-speed transmission for downsizing the motor of a fully electric vehicle," $S A E$ International Journal of Alternative Powertrains, vol. 1, no. 1, pp. 134-143, 2012.

[10] Q. Ren, D. A. Crolla, and A. Morris, "Effect of transmission design on electric vehicle (EV) performance," in Proceedings of the 2009 IEEE Vehicle Power and Propulsion Conference, pp. 1260-1265, IEEE, Dearborn, MI, USA, September 2009.

[11] J. Ruan, P. Walker, and N. Zhang, "A comparative study energy consumption and costs of battery electric vehicle transmissions," Applied Energy, vol. 165, pp. 119-134, 2016.
[12] F. C. Correa, J. J. Eckert, L. C. A. Silva, F. M. Santiciolli, E. S. Costa, and F. G. Dedini, "Study of different electric vehicle propulsion system configurations," in Proceedings of the 2015 IEEE Vehicle Power and Propulsion Conference (VPPC), pp. 1-6, Montreal, Quebec, Canada, October 2015.

[13] J. J. Eckert, L. C. A. Silva, E. S. Costa, F. M. Santiciolli, F. G. Dedini, and F. C. Corrêa, "Electric vehicle drivetrain optimisation," IET Electrical Systems in Transportation, vol. 7, no. 1, pp. 32-40, 2017.

[14] H. Qian, G. Xu, J. Yan, T. L. Lam, Y. Xu, and K. Xu, "Energy management for four-wheel independent driving vehicle," in Proceedings of the 2010 IEEE/RSJ International Conference on Intelligent Robots and Systems, pp. 5532-5537, IEEE, Taipei, Taiwan, 2010.

[15] V. Ivanov, D. Savitski, and B. Shyrokau, "A survey of traction control and antilock braking systems of full electric vehicles with individually controlled electric motors," IEEE Transactions on Vehicular Technology, vol. 64, no. 9, pp. 3878-3896, 2015.

[16] Y. Chen, X. Li, C. Wiet, and J. Wang, "Energy management and driving strategy for in-wheel motor electric ground vehicles with terrain profile preview," IEEE Transactions on Industrial Informatics, vol. 10, no. 3, pp. 1938-1947, 2014.

[17] M. Hu, J. Zeng, S. Xu, C. Fu, and D. Qin, "Efficiency study of a dual-motor coupling EV powertrain," IEEE Transactions on Vehicular Technology, vol. 64, no. 6, pp. 2252-2260, 2015.

[18] Y. Gao, W. Wang, and Y. Li, "Optimization of control strategy for dual-motor coupling propulsion system based on dynamic programming method," in Proceedings of the 2019 3rd Conference on Vehicle Control and Intelligence (CVCI), pp. 1-6, Hefei, China, September 2019.

[19] B. Wang, M. Xu, and L. Yang, "Study on the economic and environmental benefits of different EV powertrain topologies," Energy Conversion and Management, vol. 86, pp. 916926, 2014.

[20] J. Ruan and Q. Song, "A novel dual-motor two-speed direct drive battery electric vehicle drivetrain," IEEE Access, vol. 7, pp. 54330-54342, 2019.

[21] J. Kang, J. Yoo, and K. Yi, "Driving control algorithm for maneuverability, lateral stability, and rollover prevention of 4WD electric vehicles with independently driven front and rear wheels," IEEE Transactions on Vehicular Technology, vol. 60, no. 7, pp. 2987-3001, 2011.

[22] P. D. U. Coronado and H. Ahuett-Garza, "Control strategy for power distribution in dual motor propulsion system for electric vehicles," Mathematical Problems in Engineering, vol. 2015, Article ID 814307, 10 pages, 2015.

[23] B. Sun, S. Gao, C. Ma, and J. Li, "System power loss optimization of electric vehicle driven by front and rear induction motors," International Journal of Automotive Technology, vol. 19, no. 1, pp. 121-134, 2018.

[24] X. Yuan and J. Wang, "Torque distribution strategy for a front- and rear-wheel-driven electric vehicle," IEEE Transactions on Vehicular Technology, vol. 61, no. 8, pp. 3365-3374, 2012.

[25] D. Wang and B. Wang, "Research on driving force optimal distribution and fuzzy decision control system for a dualmotor electric vehicle," in Proceedings of the 2015 34th Chinese Control Conference (CCC), pp. 8146-8153, Chinese Association of Automation, Hangzhou, China, July 2015.

[26] M. Zhao, J. Shi, and C. Lin, "Energy management strategy design for dual-motor coaxial coupling propulsion electric city-buses," Energy Procedia, vol. 152, pp. 568-573, 2018. 
[27] P. Shen, Z. Zhao, X. Zhan, and J. Li, "Particle swarm optimization of driving torque demand decision based on fuel economy for plug-in hybrid electric vehicle," Energy, vol. 123, pp. 89-107, 2017.

[28] J. Ruan, P. D. Walker, P. A. Watterson, and N. Zhang, "The dynamic performance and economic benefit of a blended braking system in a multi-speed battery electric vehicle," Applied Energy, vol. 183, pp. 1240-1258, 2016.

[29] S. Amaran, N. V. Sahinidis, B. Sharda, and S. J. Bury, "Simulation optimization: a review of algorithms and applications," Annals of Operations Research, vol. 240, no. 1, pp. 351-380, 2016.

[30] H. S. Ramadan, A. F. Bendary, and S. Nagy, "Particle swarm optimization algorithm for capacitor allocation problem in distribution systems with wind turbine generators," International Journal of Electrical Power \& Energy Systems, vol. 84, pp. 143-152, 2017.

[31] M. N. Ab Wahab, S. Nefti-Meziani, and A. Atyabi, "A comprehensive review of swarm optimization algorithms," PLoS One, vol. 10, no. 5, 2015.

[32] S.-Y. Chen, Y.-H. Hung, C.-H. Wu, and S.-T. Huang, "Optimal energy management of a hybrid electric powertrain system using improved particle swarm optimization," Applied Energy, vol. 160, pp. 132-145, 2015. 